\title{
GWAS hints at pleiotropic roles for FLOWERING LOCUS T in flowering time and yield-related traits in canola
}

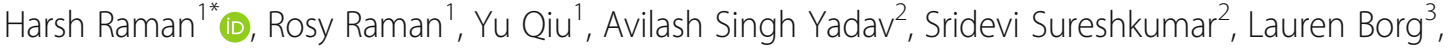 \\ Maheswaran Rohan', David Wheeler ${ }^{4}$, Oliver Owen'1, Ian Menz ${ }^{1}$ and Sureshkumar Balasubramanian²
}

\begin{abstract}
Background: Transition to flowering at the right time is critical for local adaptation and to maximize grain yield in crops. Canola is an important oilseed crop with extensive variation in flowering time among varieties. However, our understanding of underlying genes and their role in canola productivity is limited.

Results: We report our analyses of a diverse GWAS panel (300-368 accessions) of canola and identify SNPs that are significantly associated with variation in flowering time and response to photoperiod across multiple locations. We show that several of these associations map in the vicinity of FLOWERING LOCUS T (FT) paralogs and its known transcriptional regulators. Complementary QTL and eQTL mapping studies, conducted in an Australian doubled haploid population, also detected consistent genomic regions close to the $F T$ paralogs associated with flowering time and yield-related traits. FT sequences vary between accessions. Expression levels of $F T$ in plants grown in field (or under controlled environment cabinets) correlated with flowering time. We show that markers linked to the FT paralogs display association with variation in multiple traits including flowering time, plant emergence, shoot biomass and grain yield.

Conclusions: Our findings suggest that $F T$ paralogs not only control flowering time but also modulate yield-related productivity traits in canola.

Keywords: Natural variation, Flowering time, Canola, Photoperiod, genome-wide association analysis, linkage analysis, Gene expression, eQTL analysis
\end{abstract}

\section{Highlight}

The genetic association, eQTL and expression analyses suggest that $F T$ paralogs have multifaceted roles in canola flowering time, plant development and productivity traits.

\section{One sentence summary}

Paralogs of $F T$ which is known to be critical for flowering time have pleiotropic roles in yield related traits in canola.

\section{Background}

Natural variation provides a valuable resource for discovering the genetic and molecular basis of phenotypic diversity

\footnotetext{
* Correspondence: harsh.raman@dpi.nsw.gov.au

${ }^{1}$ NSW Department of Primary Industries, Wagga Wagga Agricultural Institute, Wagga Wagga, NSW 2650, Australia

Full list of author information is available at the end of the article
}

in plant development, adaptation and productivity $[1,2]$. Canola (rapeseed, Brassica napus L., $A_{n} A_{n} C_{n} C_{n}$ genomes, $2 n=4 \times=38)$ is an important oil crop, varieties of which display extensive variation in life history traits such as flowering time. Precise knowledge of flowering time is fundamental for identifying locally adapted varieties. It is also essential in the development of new varieties that maximize yield and oil quality in diverse and rapidly changing environments. For example, early flowering varieties are preferred for cultivation when periods of drought and high heat are frequent, whereas winter/semi-winter crops achieve maximum yields in the longer growing seasons that occur in temperate regions [3].

In Arabidopsis thaliana, the four major pathways that regulate flowering time are photoperiod, vernalisation, autonomous and gibberellic acid pathways [4, 5]. 
MicroRNAs, sugar status and signaling also interact with the flowering pathways to generate a complex regulatory network [6]. Flowering is also affected by other external factors such as ambient temperature, insect-pests, pathogens, light quality, and abiotic stress [1,7]. Genetic analyses based on classical linkage mapping (quantitative trait loci: QTLs) and genome-wide association studies (GWAS) have revealed that flowering time in canola is a multigenic trait [8-16]. Candidate genes underlying flowering time variation due to vernalisation have been identified in $B$. napus $[8,12,17-21]$. We have previously shown that BnFLC.A02 accounts for the majority $(\sim 23 \%)$ of variation in flowering time among diverse accessions of canola [12]. Nevertheless, little is known about functional role of the photoperiod responsive genes in modulating flowering time especially in spring canola varieties.

The gene FLOWERING LOCUS T (FT) is generally considered to integrate inputs from several pathways that finally result in floral transition. In A. thaliana, loss-offunction mutations in $F T$ result in late flowering under long-day conditions $[22,23]$. In $B$. napus, six paralogs of FT have been identified $[24,25]$ that contribute to functional divergence in flowering time between winter and spring cultivars. For example, mutations in BnC6.FTa and $B n C 6 . F T b$ paralogs have been shown to alter flowering time in B. napus accessions [26]. Owing to the multiple copies of FT in canola, it has been difficult to establish the functionality and precise relationship between various paralogs in plant development and productivity traits, as shown in Arabidopsis, onion and potato [27-32]. In addition, under field conditions, it is difficult to determine the extent of genetic variation in photoperiod response, as plants undergo a series of cold temperature-episodes during vernalisation.

Here we determine the extent of flowering time variation utilizing a diverse panel of 368 canola genotypes representing different geographic locations around the world. Using GWAS we identify several underlying QTLs controlling phenotypic variation in photoperiod response and flowering time. We show that the response to photoperiod maps to $F T$ paralogues, and their potential transcriptional regulators $C I B, C O, C R Y 2, F V E, M S I$, EMF2 and PIF4. Using a doubled haploid population of plants grown under LD and/or field conditions, we show that expression levels of $F T$ paralogs are significantly associated with flowering time variation across diverse canola accessions. The eQTL analysis for $F T$ expression levels map not only to $F T$ itself (e.g., $B n A 7 . F T$ ) but also other loci that are known regulators of $F T$ such as BnFLC.C3b (FLC5), FPA, SPA1 and ELF4. We also demonstrate that plant productivity traits such as plant emergence, shoot biomass accumulation, plant height, and grain yield map in the vicinity of FT. Taken together our findings suggest that $F T$ has multifaceted role in plants and could be exploited for selection of canola varieties for improved productivity.

\section{Materials and methods \\ Plant material and growth conditions Evaluation of GWAS panel}

A diverse panel of 368 accessions of B. napus L. was used to evaluate photoperiod response in this study (Additional file 1: Table S1). A 300 accessions subset of these was evaluated for flowering time in three field experiments: (a) in plots $\left(35^{\circ} 03^{\prime} 36.9^{\prime \prime} \mathrm{S} 147^{\circ} 18^{\prime}\right.$ $40.2^{\prime \prime} \mathrm{E}, 147 \mathrm{~m}$ above sea level) at the Wagga Wagga Agricultural Institute (WWAI) located at Wagga Wagga, NSW, Australia, (b) in plots at the Condobolin Agricultural Research and Advisory Station, NSW, Australia (33. $0418.98^{\circ} \mathrm{S}, 147.1350 .16^{\circ} \mathrm{E}, 220 \mathrm{~m}$ above sea level) and (c) in single rows at WWAI $\left(35^{\circ} 02^{\prime} 27.0^{\prime \prime} \mathrm{S} 147^{\circ} 19^{\prime} 12.6^{\prime \prime} \mathrm{E}\right)$ in 2017 canola growing season. For WWAI plot trial, 300 accessions were arranged in a randomized complete block design with 60 rows by 10 columns (ranges) in four flood irrigation bays, each bay had 15 rows and 10 ranges (Additional file 2: Table S2). A buffer (non-experimental line) row of an Australian canola variety SturtTT was seeded after every two ranges to ensure that plots are harvested at the right maturity. For WWAI single row trial, 300 accessions were arranged in a randomized block design with 60 rows (each row $10 \mathrm{M}$ long) by 10 columns in two replicates (Additional file 2: Table S2), each replicate of 30 rows was separated with a buffer row of SturtTT. Each accession per replication had 100 plants. This trial was sown under Lateral Move irrigation system to match water demand for optimal plant growth. The Condobolin trial was sown as rainfed and arranged in a randomised complete block design with 100 rows by 6 columns, accommodating all 300 accessions in two replicates (Additional file 2: Table S2). For field plot experiments, accessions were sown in plots ( $2 \mathrm{~m}$ wide $\times 10 \mathrm{~m}$ long at Wagga Wagga and $2 \mathrm{~m}$ wide $\times 12 \mathrm{~m}$ long at Condobolin) at density of 1400 seeds $/ 20 \mathrm{~m}^{2}$ plot. Seeds were counted with Kimseed machine and directly sown in plots in the field; each plot consisted of 6 rows spaced $25 \mathrm{~cm}$ apart. Plots were sown with a six-row cone-seeder to $10 \mathrm{~m}$ length. All plots were sown with a granular fertilizer (N: P: K: S, 22: 1: 0: 15) applied at $150 \mathrm{~kg} \mathrm{ha}^{-\mathrm{P}}$. The fertilizer was treated with the fungicide Jubilee (a.i. flutriafol at $250 \mathrm{~g} / \mathrm{L}$, Farmoz Pty Ltd., St Leonards, NSW, Australia) to protect all genotypes against the blackleg fungus, Leptosphaeria maculans. After crop establishment, plots were trimmed back to $8 \mathrm{~m}$ after emergence by applying Roundup (a. i. glyphosate) herbicide with a shielded spray boom. For controlled environmental cabinets (CE cabinets, Thermoline Scientific, Wetherill Park NSW, Australia), eight plants of each of the 368 accessions were grown in plastic trays as described previously [12] under long (LD) and short 
day (SD) conditions. For LD treatment, seeds were planted in a $\mathrm{CE}$ maintained at $20 \pm 1{ }^{\circ} \mathrm{C}$ under white fluorescent lamps (4000 K, Osram) with light intensity of approximately $150 \mu \mathrm{M} / \mathrm{m}^{2} / \mathrm{s}$, with a $16-\mathrm{h}$ photoperiod. In SD treatment, plants from 368 accessions were grown at the same conditions described above but for $8 \mathrm{~h}$ photoperiod.

\section{Flowering time and other phenotypic measurements}

Days to flower from sowing was calculated when $50 \%$ of plants had opened their first flower. In SD conditions, flowering time was recorded for up to 200 days. Plants without any flowers at the end of the experiments were classified as 'assigned (A)' (LD-A, SD-A, see Fig. 1). The response to photoperiod was calculated as the difference between $50 \%$ flowering in plants grown under SD and LD conditions. For field trials, flowering time was recorded three times in a week.

Normalised Difference Vegetative Index (NDVI) was measured as a proxy of fractional ground cover for early vigour [33, 34] using a GreenSeeker (model 505, NTech Industries Inc., Ukiah, CA, USA). The NDVI readings

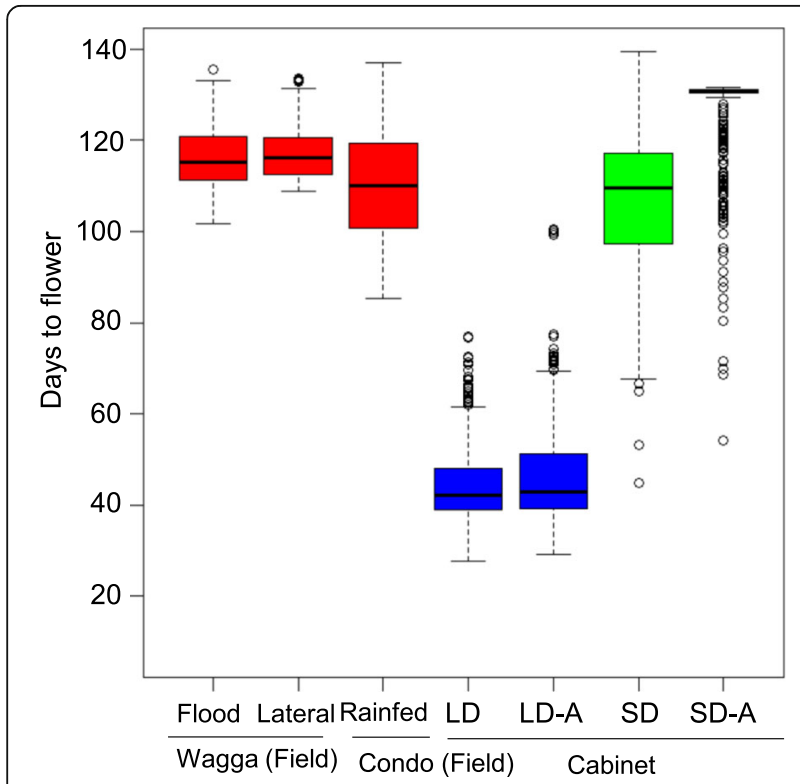

Fig. 1 Natural variation for flowering time. Box-plots showing genetic variation for flowering time in a diverse panel of canola accessions, which were grown across five experiments under field, and controlled environment conditions (cabinets). Days to flowering were scored in 2017: Field plots at Wagga Wagga (flood irrigated) and Condobolin (Condo, rainfed); Single rows at Wagga Wagga (lateral move irrigation); Days to flowering were scored in long day condition (LD, 16 h) and short day condition (SD, 8 h) plants under cabinets. Genotypes that did not flower till the end of experiment were also included and marked as flowering 'assigned' (LD-A and SD-A). A total of 368 accessions were evaluated for flowering time under LD and SD conditions, while 300 accessions were evaluated under field conditions. Details are given in Additional file 1: Table S1 were taken at 7-10 days interval after 5 weeks of sowing before the onset of flowering. Multiple readings were taken in each plot and then averaged across each plot for genetic analysis. Plots were harvested by direct heading with a Kingroy plot harvester (Kingaroy Engineering Works, Queensland, Australia) in the 4th wk. of November (Condobolin, NSW) and 2-3rd wk. of December (Wagga, Australia). Grain samples were cleaned with Kimseed (Kimseed Australia, Western Australia) and plot yield was expressed into t/ha.

\section{Field evaluation of SAgS DH population}

SAgS population of $144 \mathrm{DH}$ progeny from a $\mathrm{BC}_{1} \mathrm{~F}_{1}$ plant derived from the cross Skipton (less responsive to vernalisation) and Ag-Spectrum (more responsive to vernalisation) have been previously described [12, 13, 35]). The population was grown in $2015\left(35^{\circ} 01^{\prime} 32.3^{\prime \prime} \mathrm{S}\right.$ $\left.147^{\circ} 19^{\prime} 25.4^{\prime \prime} \mathrm{E}\right)$ and $2016\left(35^{\circ} 01^{\prime} 42.8^{\prime \prime} \mathrm{S}, 147^{\circ} 20^{\prime} 23.3^{\prime \prime} \mathrm{E}\right)$ in the field at the WWAI, NSW, Australia. Both trials were randomised in a complete block design with three replicates in a single block. A total of 1,400 seeds per genotype were directly sown in plots in the field as described above. The traits measured included plant emergence, first flowering, plant biomass, plant height, and grain yield. Plant (shoot) biomass was calculated from cuttings obtained from 10 randomly selected plants growing in the central row of each plot. Each sample was weighed on a digital scale and fresh weights were expressed in $\mathrm{g} /$ plant. Plant height $(\mathrm{cm})$ was measured at the physiological maturity stage using 5 plants selected randomly in the middle row of each plot. Plots were harvested with a Kingaroy plot harvester in the $2-3$ rd wk. of December (Wagga, Australia).

\section{Genome-wide genotyping}

Leaf material was collected individually from 368 diverse DH canola accessions, grown under LD conditions, and then immediately snap-frozen in liquid nitrogen. Genomic DNA was isolated as described previously [13] and sent to Trait Genetics, Germany (http://www.traitgenetics.com/) for genotyping with Illumina infinium $15 \mathrm{k}$ Brassica chip representing $60 \mathrm{~K}$ Infinium SNP array [36].

\section{Population structure and GWA analyses}

For GWA analysis, we only used SNP markers with allele frequencies $>0.05$ and overall call rates (proportion of genotypes per marker) of $>90 \%$ [37]. To prevent the potential loss of genome wide associations (GWA) missing data was imputed [38]. A total of 11,804 SNP markers could be anchored to the $A_{n}$ and $C_{n}$ subgenomes of reference sequenced genome of $B$. napus $c v$. The variety 'Darmor- $b z h$ ' (Darmor) was used as reference for cluster and GWA analyses in a diversity panel of 368 accessions (Additional file 1: Table S1). 
Cluster analysis was performed with the NeighborJoining method [39] using MEGA version 6. In order to reduce spurious associations between markers and variation in flowering time, population structure and the relative kinship coefficients of individual genotypes were estimated as described previously [12]. Flowering time-SNP marker association analysis was performed using the EMMAx/P3D method [40, 41] implemented in the $\mathrm{R}$ package GAPIT [42] (https:// cran.r-project.org/). Significance of GWA between markers and flowering time was tested at LOD score of 3 . The $P\left(-\log _{10} P\right)$ values for each SNP were exported to generate a Manhattan plot in R [43]. The proximity of candidate genes to identified associations based on the physical positions of SNPs/candidate genes was inferred based on functional annotation of the $A$. thaliana genome and implemented in the reference sequenced genome of Darmor [44]. After Bonferroni correction, associations with LOD score $=5.41$ were also considered as significant on a $p<0.05$ level. The associations detected through GWAS were compared with the QTL intervals associated with flowering time identified in the field conditions in the SAgS DH mapping population evaluated in 2013, 2014 [13], 2015 and 2016 (this study).

\section{Statistical and QTL analysis}

Flowering and other phenotypic data collected from different experiments were analysed using linear mixed models in R as described previously [45]. Essentially, we defined the individual experimental Plot as a factor with 432 levels for each of the 2015 and 2016 trials. The factors: Row and Range corresponded to the rows and ranges of the trials, with levels equal to the number of rows and ranges in each trial. The combination of levels of Row and Range completely index the levels of Plot such that Plot $=$ Row:Range. The factor Rep has 3 levels corresponding to the replicate blocks in each trial. The plot structure for the field experiment consists of plots nested within blocks and is given by, Rep/Plot which can be expanded to give, Rep + Rep:Plot. The term Rep:Plot indexes the observational units for all traits and thus is equivalent to the residual term for these traits. The treatments for the field phase of the experiment are the lines allocated to plots so we define the treatment factor, Genotype, with 144 levels corresponding to lines grown in each trial. Due to marker data being included in the model, we need to define an additional two factors; Gkeep (corresponding to lines with both phenotypic and marker data) and Gdrop. The factor Gdrop has 16 levels corresponding to lines with phenotypic data but not marker data. Therefore treatment structure is given by Gkeep + Gdrop. Finally, marker data is incorporated into the analysis and individual markers are scanned following the approach of Nelson et al. (2014) [9] to establish a final multi-QTL model. We also used phenotypic data from 2013 and 2014 experiments that was published previously [13], in order to test multifaceted role of $F T$ in flowering time and other productivity traits across environments. A genetic map based on 7,716 DArTseq markers representing 499 unique loci [13] was used to determine trait-marker associations. The predicted means for first flowering, and response to photoperiod for each genotype were used to detect genome wide trait-marker associations.

\section{FT expression and eQTL analyses}

$F T$ expression analysis was carried out in two different sets of populations. First, we analysed $F T$ expression in field-grown plants from $144 \mathrm{DH}$ lines of the SAgS DH population. Second, we analysed $F T$ expression in 24 accessions that represented extreme flowering phenotypes (i.e., early and late flowering accessions) from the 368 accessions in the GWAS panel. For both sets of experiments, five independent leaf samples collected from field/CE grown plants (at floral budding stage) per genotype were pooled and flash-frozen in liquid nitrogen (in field/ $\mathrm{CE})$. For field-grown plants there were internal replications that effectively represented at least two biological replicates. For CE grown plants three biological replicates were used. RNA was isolated using TRIZol (Invitrogen) and cDNA was synthesized using a First Strand Synthesis Kit (Roche). Samples were controlled for their quality using two approaches as outlined previously [12]. Gene specific primers for each of six $F T$ paralogs [26] used for the expression analysis are described in Additional file 3: Table S3. Since the expression levels of all $F T$ paralogs were correlated, we used data from $B n C 6 . F T$ for $\mathrm{eQTL}$ analysis using SVS package (Golden Helix, Bozeman, USA).

\section{Structural variation in canola $F T$ paralogs}

We obtained sequence information for $F T$ paralogs from a whole-genome resequencing data for the 21 canola accessions, which will be described elsewhere (Raman et al., in preparation). These 21 accessions also included the parental lines (Skipton and Ag-Spectrum) of the SAgS mapping population used in this study (Additional file 2: Table S2). Variation across the $F T$ paralogs was extracted using the gene model information or by manually identifying gene regions based on BLAT homology (Additional file 4: Table S4). The physical positions of different $F T$ paralogs (NCBI GenBank accessions; genomic sequences: FJ848913 to FJ848918; promoter sequences: JX193765, JX193766, JX193767, JX193768) were confirmed with those of the sequenced FT genes on the 'Darmor' assembly as well as with published literature $[24,25,46]$. For each accession, the $F T$ nucleotide sequences were aligned using MUSCLE as implemented [47] in the software package Geneious (https://www.geneious.com) Structural variation, number 
of polymorphic sites within the gene and the promoter region was identified using ANNOVAR [48]. The diversity indices were calculated using the MEGA version 6 [49]. The Tajima [50] and Fay and Wu [51] tests were conducted to examine whether the frequency spectrum of polymorphic nucleotide mutations conformed to the expectations of the standard neutral model. The effect of InDel mutations on functional domains was investigated using information from the NCBI conserved domain database.

\section{Results}

\section{Natural variation in flowering time across diverse environments}

We determined the natural variation in flowering time of diverse accessions across five different environments. Across all environmental conditions, we found extensive variation in flowering time, which ranged from as little as 29.2 days up to more than 137 days (Fig. 1, Additional file 5: Table S5 and Additional file 6: Table S6). Diverse accessions grown under LD conditions (16 h light at $20^{\circ} \mathrm{C}$ ) typically flowered earlier (29.2 to 100.6 days) than those grown in either SD (54.3 to 131.5 days under $8 \mathrm{~h}$ light at $20{ }^{\circ} \mathrm{C}$ in growth cabinet) or field conditions (85.2 to 137.1 days). Accessions grown under rainfed conditions (Condobolin site) flowered earlier compared to those grown at the irrigated Wagga Wagga sites (Additional file 6: Table S6). Most of this variation was genetically controlled as the broad sense heritability $\left(h^{2}\right.$, also called as reliability) ranged from 45 to $97 \%$ across different environments (Additional file 7 : Table S7). We observed positive genetic correlations ( $r=0.88$ to 0.96 ) for flowering time between the different field trials, suggesting that majority of the genetic variation and underlying mechanisms are shared across environments (Fig. 2).

\section{Flowering time variation in canola is largely due to photoperiodic response}

Under controlled environmental conditions in growth cabinets, LD photoperiod substantially promoted flowering (27.6 to 77 days) (Additional file 5: Table S5, Fig. 1), while only $23.8 \%$ of accessions $(n=86)$ flowered under short days, suggesting that extended photoperiod is required for flowering. Analysis of photoperiodic response in accessions enabled us to identify specific accessions of interest, with robust photoperiod sensitive or insensitive behavior (Fig. 1, Additional file 5: Table S5). Only a small proportion $(6.25 \%, n=23)$ of accessions did not flower within 100 days under LD conditions. None of the winter type accessions (e.g., 03-P74, Azuma, Beluga, Ding10, Erglu, FAN28, FAN168, Gundula, Haya, HZAU-1, Maxol, Primor, Rangi, Norin-19, Tower, ZY002, ZY14, Zhongshuang-4, Zhongyou 8) flowered either in LD or in SD condition, reconfirming that vernalisation is essential for flowering in those accessions. This is consistent with these genotypes being winter/semi-winter types that typically require vernalisation to flower [12]. To assess whether there is any differential photoperiodic response, we compared the effects of photoperiod on flowering time of the accessions grown in controlled environment cabinets. Four accessions, 9X360-310 (BC15278), Georgie (BC15289), CB-Tanami (BC52411) and Hylite200TT (BC52662) had atypical flowering response, suggesting genotype $\mathrm{x}$ environment interactions (Additional file 1: Table S1b, Additional file 19: Figure S1).

\section{Relationship between flowering time and other traits}

To determine whether there is any relationship between flowering time and yield-related traits in canola, we analysed their genetic correlations (Fig. 3). There were low genetic correlations between the flowering time and other agronomic traits, which suggests that the growth environment play an important role in trait expression. Flowering time showed a negative correlation with grain yield across sites (WW-Wagga Wagga and Con: Condobolin) under LD photoperiodic conditions (field and controlled environments). Early vigour (NDVI.WW) showed positive correlations with flowering time $(r=0.2$ to 0.7) under LD and field conditions (WW-Wagga and Con), and with grain yield ( $r=0.1$ to 0.4 ) depending upon growing environment.

Genetic relatedness among accessions in the GWAS panel SNP marker distribution across genome is shown in Additional file 20: Figure S2. SNP markers were distributed un-evenly: most were located on chromosomes A03, A07, C03, and C04 (> 780 markers/chromosome). The lowest marker density was observed in chromosome C09. A total of 11,804 SNP markers anchored to the reference $B$. napus genome, with the mean marker density of 621.3 per chromosome provided coverage of $\sim 84.7$ $\mathrm{kb} /$ marker. Cluster analysis revealed at least three main clades among accessions, representing European winter, Australian semi-spring/Canadian spring, and semi-winter of Indian/Chinese origin (Fig. 4, Additional file 21: Figure S3). The first three principal components $(\mathrm{PC} 1=38.1 \%, \mathrm{PC} 2=$ $11.9 \%$, and $\mathrm{PC} 3=5.67 \%$ ) accounted for $55.7 \%$ of the genetic variation and the grouping of accessions reflected the cluster analysis (Additional file 22: Figure. S4). To estimate the extent of genome-wide linkage disequilibrium (LD) we calculated the squared allele frequency correlations (average $r^{2}$ ) for all pairs of the anchored SNPs using an LD sliding window of 500 as 0.02 (Additional file 23: Figure S5). The kinship coefficient among accessions ranged from 0.03 to 0.99 suggesting a wide-range of familial relatedness between pairs of accessions (Additional file 8: Table S8), as observed in our previous study [12]. 
a

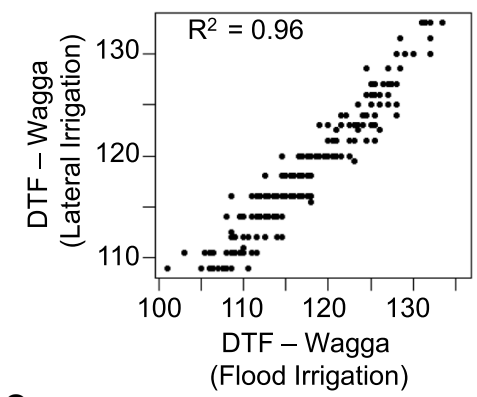

C

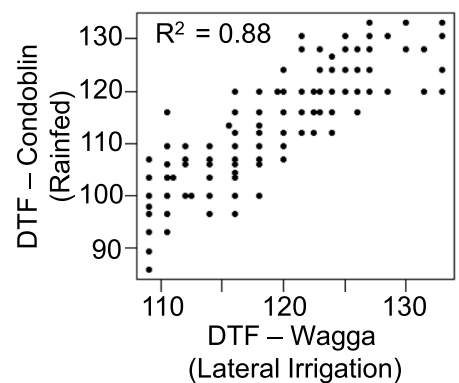

b

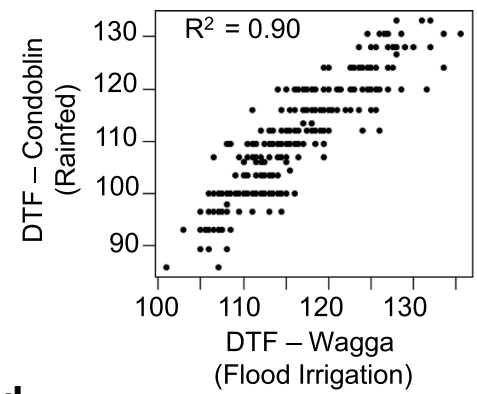

d

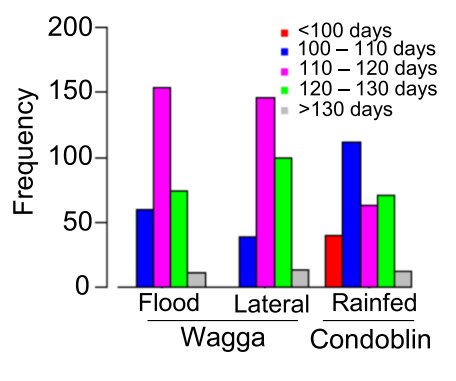

Fig. 2 Pearson correlation for flowering time among 300 accessions of canola evaluated in field plots across different environments. Flowering time (days to flower, DTF) was assessed thrice in a week. a Flowering time correlation between field trials that were irrigated with lateral move or via flooding. b Flowering time correlation between field trials at flood irrigated plots at Wagga with rainfed plots at Condobolin. c Flowering time correlation between laterally irrigated plots at Wagga and rainfed plots at Condobolin and $\mathbf{d}$ Frequency distribution of canola accessions based on the days to first flower under the varied conditions

\section{Genetic architecture of flowering time and photoperiod response}

Accounting for both population structure and kinship information, we detected a total of 142 significant associations (at the genome-wide significance thresholds of LOD score of $\geq 3$ ) for flowering time in diverse environments [(under field, three experiments), LD and SD conditions)]. The markers with significant associations were distributed across all chromosomes except A01 (Additional file 9: Table S9). Majority of the associated SNPs (70\%) were identified on $A_{n}$ subgenome (Additional file 10: Table S10), suggestive of an uneven distribution on the physical locations of Darmor assembly. Most of the associated SNPs (33.1\%) were on chromosome A02 (47 SNPs), followed by $9.15 \%$ on C03 (13 SNPs), and these could explain the majority of allelic variation for flowering time in canola. We identified 22 unique SNP markers that accounted for associations that were detected at least in 2 different environments (Additional file 9: Table S9). Of the 142 significant associations, six SNPs crossed the Bonferroni threshold for flowering time in LD conditions, all of which are located on chromosome A02 (Table 1). Two of these SNPs (Bn-A02-p9371948 and Bn-A02-p9371633) associated with flowering time under $\mathrm{LD}$ conditions were located near the FT locus $(\sim 0.64 \mathrm{Mb}$, BnA02.FT,
BnaA02g12130D) (Fig. 5a-c). Under different environmental conditions, we detected different associations; several of these SNP associations were mapped near the vicinity of genes known to play a regulatory role in $F T$ expression in A. thaliana such as FLC4, UPSTREAM OF FLC, CO, MSI1, LD, MAF4 on A02; BnFLC3a, CO and EMF2 on A03; $N Y-Y B 8$ on A04; GI on A08; EMF2 and CRY2 on A10, and CIB1 on C08 (Additional file 11: Table S11). We also identified 28 SNPs that showed significant association above a LOD of 3 with response to photoperiod identified under controlled environment cabinet conditions on chromosomes A01, A02, A07, A09, A10, C01, C03, C06, C08 and C09 (Additional file 11: Table S11, Fig. 5c).

To identify potential candidates involved in the photoperiod response, we compared the physical positions of 28 significant SNP associations for photoperiod with the physical positions of flowering time genes (Additional file 11: Table S11). Seven significantly associated SNP markers map in the vicinity $(0.2 \mathrm{Mb})$ of SPA3 (A01), PRR5 (A02), MAF4 (A02), ASH1 (A07), POWERDRESS (A10) and ELF6 (C09), genes underlying photoperiod response in canola accessions. The genes ANAC029, EFF6, $A B F 2, F V E$, and $P A F 1$ were also identified in CE experiments and $A N A C 029$, and $A S H 1$, were also identified under field experimental conditions (Additional file 24: 


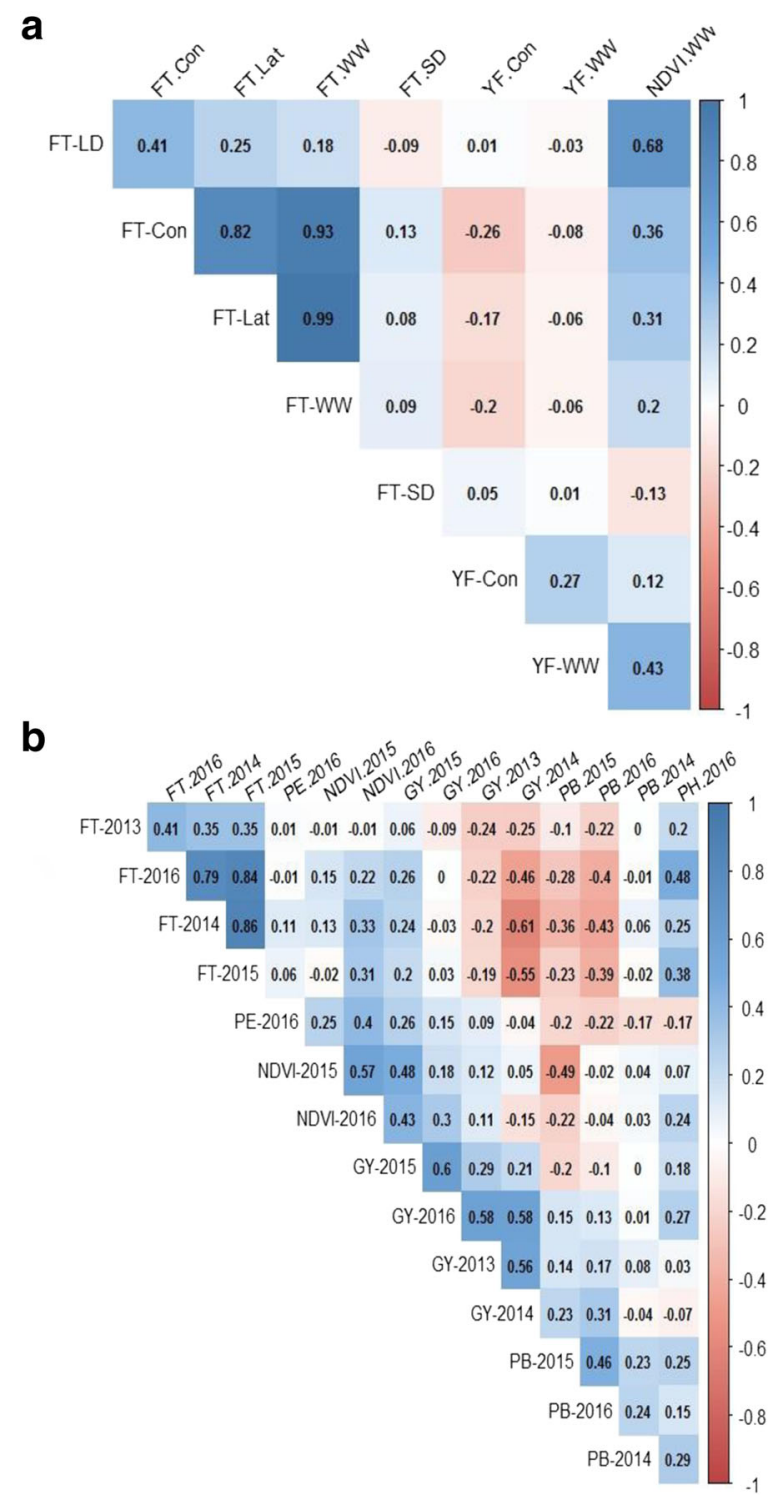

Fig. 3 Pearson correlation between flowering time (FT) and yield related traits in a GWAS panel (a) and DH population derived from Skipton/Ag-Spectrum//Skipton (b). FT-LD: flowering time under LD conditions (days to flower); FT-SD: flowering time under SD conditions (days to flower); FT-Con: flowering time at Condobolin; FT-Lat: flowering time at Wagga (lateral move); FT-WW: flowering time at Wagga (rainfed); YF-Con: Grain yield at Condobolin; YF-WW: Grain yield at Wagga (flood irrigation), NDVI.WW: Normalised Difference Vegetative Index at Wagga; PE: plant emergence; GY: grain yield; PB: plant biomass ( $\mathrm{g} /$ plant) and PH: plant height $(\mathrm{cm})$

Figure S6; Additional file 11: Table S11). Consistent with our previous study (Raman et al. 2016a), our results reinforces that while the major players of flowering time appear to be conserved between Arabidopsis and canola, the specific functional roles of the paralogs might differ depending on the environmental conditions.

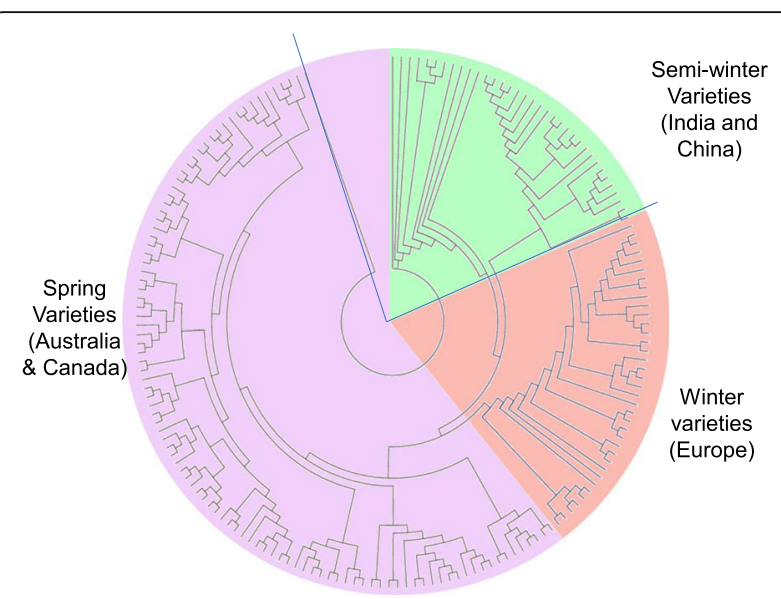

Fig. 4 Molecular diversity in a GWAS panel of 368 Brassica napus accessions. Three dominant clusters shown in different shades; violet, red and light green colors represent predominantly spring, winter and semi-winter accessions of Australian, European, and IndoChinese origins, respectively. Details are given in Additional file 1: Table S1. Tree was drawn with MEGA 6 package [48]

\section{QTL analysis in biparental population identifies loci for} flowering time and productivity traits near FT paralogs To ensure capturing the relevance of entire genetic architecture of flowering time variation, we considered the SAgS DH mapping population derived from a $\mathrm{BC}_{1} \mathrm{~F}_{1}$ cross between Australian spring type cultivars; Skipton (less responsive to vernalisation) and Ag-Spectrum (more responsive to vernalisation). We had previously utilised this cross for genetic analyses for range of traits of interest $[8,13,35,52-54]$. The frequency distributions of the $\mathrm{DH}$ lines for different traits evaluated are shown (Additional file 25: Figure S7). The DH lines exhibited high broad sense heritability values (56.7 to 99\%) for all traits, except for NDVI and plant emergence (29.2 to $44.3 \%$ ) across environments (Additional file 12: Table $\mathrm{S} 12 \mathrm{a})$. There was moderate to high genetic correlations for flowering time, early vigour, plant biomass and grain yield across environments (phenotyping years) in the SAgS DH population (Fig. 6). Flowering time showed generally negative correlations with grain yield and plant biomass, whereas it showed positive correlation with early vigour and plant height. We identified several QTLs associated with flowering time, plant emergence, shoot biomass, plant height, and grain yield across phenotypic environments in the SAgS population (Additional file 12: Table S12b).

Since we detected moderate to high genetic correlations in this population between multiple traits including flowering time (Additional file 13: Table S13), we considered whether the QTLs underlying these multiple phenotypes co-localise onto the physical map of $B$. napus. Genetic and physical localisation of markers on 
Table 1 Genome-wide highly significant SNPs associated with variation in flowering time and photoperiodic response in diverse accessions of $B$. napus. Photoperiod response was evaluated under long (LD) and short day (SD) conditions in the controlled environment cabinet (CE). QTL marked with * were detected in the SAgS (Skipton/Ag-Spectrum/Skipton) DH population (Raman et al. 2013 [8], $2016[12,13,35])$

\begin{tabular}{|c|c|c|c|c|c|c|c|c|c|}
\hline $\begin{array}{l}\text { Growth } \\
\text { Condition }\end{array}$ & $\begin{array}{l}\text { Experiment } \\
\text { site }\end{array}$ & SNP & Chromosome & $\begin{array}{l}\text { Physical } \\
\text { Position on } \\
\text { B. napus cv. } \\
\text { Darmor } \\
\text { assembly }\end{array}$ & $\begin{array}{l}P \text {. value } \\
\text { for genetic } \\
\text { association }\end{array}$ & $R^{2}(\%)$ & $\begin{array}{l}\text { Physical } \\
\text { Distance } \\
\text { from } \\
\text { candidate } \\
\text { gene (Mb) }\end{array}$ & $\begin{array}{l}\text { Putative } \\
\text { Candidate } \\
\text { gene }\end{array}$ & $\begin{array}{l}\text { Other flowering } \\
\text { time QTL found } \\
\text { within } 200 \mathrm{~Kb} \\
\text { regions }\end{array}$ \\
\hline $\mathrm{LD}$ (CE) & $\begin{array}{l}\text { Wagga } \\
\text { Wagga }\end{array}$ & $\begin{array}{l}\text { Bn-A02- } \\
\text { p1232964 }\end{array}$ & $\mathrm{A} 02$ & 147990 & $5.32 \mathrm{E}-07$ & 4.005696 & 0.014152 & $\begin{array}{l}\text { UPSTREAM } \\
\text { OF FLC }\end{array}$ & Wagga (Field) \\
\hline $\mathrm{SD}(\mathrm{CE})$ & $\begin{array}{l}\text { Wagga } \\
\text { Wagga }\end{array}$ & $\begin{array}{l}\text { Bn-A02- } \\
\text { p1232964 }\end{array}$ & A02 & 147990 & $1.13 \mathrm{E}-06$ & 6.162398 & 0.014152 & $\begin{array}{l}\text { UPSTREAM } \\
\text { OF FLC }\end{array}$ & Wagga (Field) \\
\hline $\begin{array}{l}\text { Field } \\
\text { (plots) }\end{array}$ & Condobolin & $\begin{array}{l}\text { Bn-A02- } \\
\text { p1232964 }\end{array}$ & A02 & 147990 & $1.25 \mathrm{E}-06$ & 6.558129 & 0.014152 & $\begin{array}{l}\text { UPSTREAM } \\
\text { OF FLC }\end{array}$ & Wagga (Field) \\
\hline LD (CE) & $\begin{array}{l}\text { Wagga } \\
\text { Wagga }\end{array}$ & $\begin{array}{l}\text { Bn-A02- } \\
\text { p10020231 }\end{array}$ & A02 & 6858767 & 4.01E-07 & 4.096034 & 0.482858 & FT (BnaA02g12130D) & $\begin{array}{l}\text { *DTF-RV (GH), } \\
\text { Biomass } 2015 \\
\text { (SAgS DH), Qdtf } \\
\text { (f/s).wwai-A2a- } \\
\text { SAgS DH }\end{array}$ \\
\hline LD (CE) & Wagga Wagga & $\begin{array}{l}\text { Bn-A02- } \\
\text { p10096185 }\end{array}$ & $\mathrm{A} 02$ & 6922499 & $1.47 \mathrm{E}-06$ & 3.683964 & 0.54659 & FT (BnaA02g12130D) & $\begin{array}{l}\text { *DTF-RV (GH), } \\
\text { Biomass 2015 } \\
\text { (SAgS DH), Qdtf } \\
\text { (f/s).wwai-A2a- } \\
\text { SAgS DH }\end{array}$ \\
\hline LD (CE) & $\begin{array}{l}\text { Wagga } \\
\text { Wagga }\end{array}$ & $\begin{array}{l}\text { Bn-A02- } \\
\text { p10176579 }\end{array}$ & $\mathrm{A} 02$ & 7019192 & $2.48 \mathrm{E}-09$ & 5.754962 & 0.643227 & FT (BnaA02g12130D) & $\begin{array}{l}\text { *DTF-RV (GH), } \\
\text { Biomass 2015 } \\
\text { (SAgS DH), Qdtf } \\
\text { (f/s).wwai-A2a- } \\
\text { SAgS DH }\end{array}$ \\
\hline LD (CE) & $\begin{array}{l}\text { Wagga } \\
\text { Wagga }\end{array}$ & $\begin{array}{l}\text { Bn-A02- } \\
\text { p10485644 }\end{array}$ & A02 & 7344509 & 7.38E-07 & 3.901669 & 0.525739 & RAV2 & $\mathrm{LD}(\mathrm{CE})$ \\
\hline LD (CE) & $\begin{array}{l}\text { Wagga } \\
\text { Wagga }\end{array}$ & $\begin{array}{l}\text { Bn-A02- } \\
\text { p10493685 }\end{array}$ & A02 & 7351405 & $2.34 \mathrm{E}-06$ & 3.536863 & 0.519263 & RAV2 & $\mathrm{LD}(\mathrm{CE})$ \\
\hline $\begin{array}{l}\text { Field } \\
\text { (single } \\
\text { row) }\end{array}$ & $\begin{array}{l}\text { Wagga } \\
\text { Wagga }\end{array}$ & $\begin{array}{l}\text { Bn-A03- } \\
\text { p471570 }\end{array}$ & A03 & 373818 & & 6.217928 & 0.140957 & TFL1 & $\begin{array}{l}\text { Field (single row) } \\
\text { Field plots }\end{array}$ \\
\hline
\end{tabular}

Darmor reference genome [44] revealed that three significant, co-located, QTLs associated with multiple traits (Fig. 7). A multi-trait QTL flanked by markers 3110489 and 3075574 for plant emergence, shoot biomass, flowering time, and grain yield mapped on chromosomes A07 was located within $0.65 \mathrm{Mb}$ of the FLOWERING LOCUS T (FT, NCBI accession FJ848914.1); $B n A 2 . F T$ paralog in B. napus [24]. Consistent with GWAS analysis, we detected QTLs near the FT in the biparental population (Fig. 7). Mapping of pleiotropic trait QTL in the vicinity of $F T$ (A07) suggest that $F T$ may have multifaceted role in plant development and productivity traits.

\section{Expression levels of $F T$ paralogs explain significant variation in flowering time}

To assess whether changes in the expression of different FT paralogs could explain the phenotypic variation in flowering time, we examined expression of FT paralogs among field-grown plants of all $144 \mathrm{DH}$ lines. Expression levels of all 6 FT paralogs displayed significant association with flowering time $(p<0.001)$, with different copies accounting for genetic variation in flowering time variably; ranging from $23 \%(B n C 2 . F T)$ to $40 \%$ (BnC6.FTb) (Fig. 8a). The FT homologues BnA7.FTb and BnA7.FTa localised near a multiple trait QTL (Additional file 12: Table S12) could explain 30 and $31 \%$ of genetic variation in flowering time, respectively. Sequence analyses of the PCR products also confirmed that $B n C 6 . F T b$ and $B n A 7 . F T b$ are accurately detected in our assays.

To further assess whether a similar pattern is also observed among natural variants, we assessed the expression of BnC6.FTb, BnA2.FT2 and BnFLC.A02. We choose $B n C 6 . F T b$ because it showed the highest correlation in the DH population. BnA2.FT2 was detected as a QTL in the diversity set of 24 accessions, whilst BnFLC.A02 was identified in accessions that differed significantly in their flowering time. Consistent with the QTL analysis and the expression studies in $\mathrm{DH}$ populations, we observed 


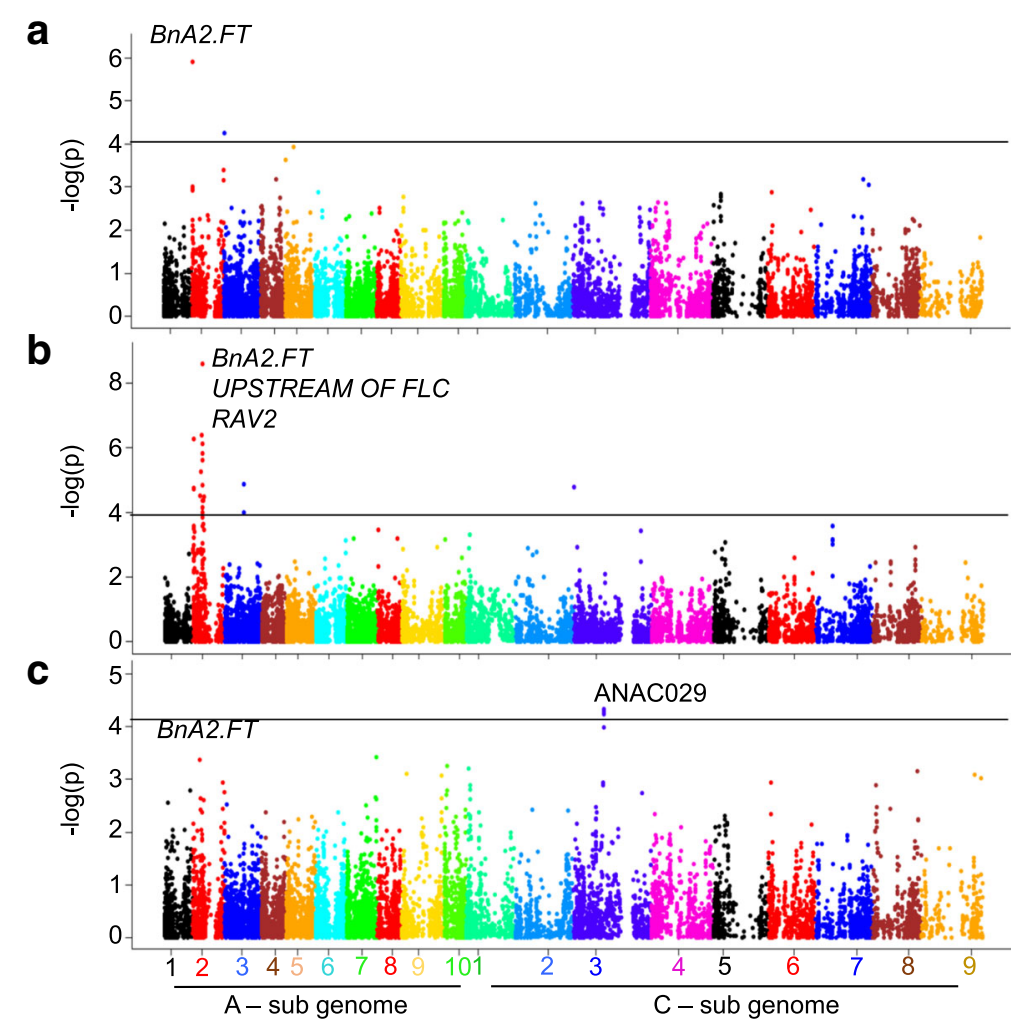

Fig. 5 Manhattan plots for the detected associations for flowering time. Plots show genome-wide $P$ values for associations between SNP markers and flowering time: a Field condition at Condobolin, Australia, $\mathbf{b}$ long-day conditions in controlled environnent cabinet and $\mathbf{c}$ response to photoperiod. Different colors represent different chromosomes of B. napus (A1-A10, C1-C9). The solid horizontal line (in black colour) signifies the threshold for significant associations - $\log 10(p)$ value of $\leq 4$

significant differences in FT and FLC expression that correlated with flowering time among 24 diverse accessions selected on the basis of flowering time diversity (Fig. 8b). Consistent with the timing of sample collection (i.e., just prior to flowering), we detected expression variation in FT rather than FLC accounting for most of the flowering time variation in these diverse set of 24 accessions. Taken together this study revealed that irrespective of the causal variation, the phenotypic variation is associated with changes in the expression levels of the floral integrator $F T$.

To unravel the cis and trans acting candidates associated with differential $F T$ transcripts expression, we first sought SNPs that affect expression levels of all FT homologues in diverse canola accessions. Then, we layered this information on the physical map positions of SNPs associated with genetic variation in flowering time and photoperiod response (Additional file 14: Table S14). We identified a total of 13 SNPs mapped on chromosome A07 and C03, in the vicinity of multiple trait QTLs that we identified in the SAgS population. The candidate genes located near significant SNP associations are FT,
ELF4-L2, PRR9, VIN3, BnFLC.C3b (FLC5, AY036892.1), FPA, SPA1 and TOE1 (Additional file 11: Table S11).

\section{FT paralogs exhibit structural sequence variation in $B$.} napus accessions

In total, nine FT copies were identified in B. napus accessions (Additional file 15: Table S15), including three putative FT copies on chromosomes A01, C02, and C04, (Additional file 15: Table S15). Sequence analyses showed considerable variation in level of synonymous and nonsynonymous SNP variations, Insertion-deletions (InDel) in promoters, as well as exonic and intronic regions. A total of 310 segregating sites were detected across FT paralogs. Our results showed that frequency spectrum of structural variants for BnA02.FT, BnC02.FT and BnC06.FT conformed to neutral expectations, while BnC04.FT and BnA07.FT showed non-conformance to neutrality, suggesting evidence of selection (Additional file 16: Table S16). We detected high level of diversity in FT paralogs mapped on A07, C04 and C06 chromosomes (Additional file 17: Table S17, Additional file 18: Table S18). For example, BnC04.FT (BnaC04g14850D) contained 35 SNPs, with the majority 

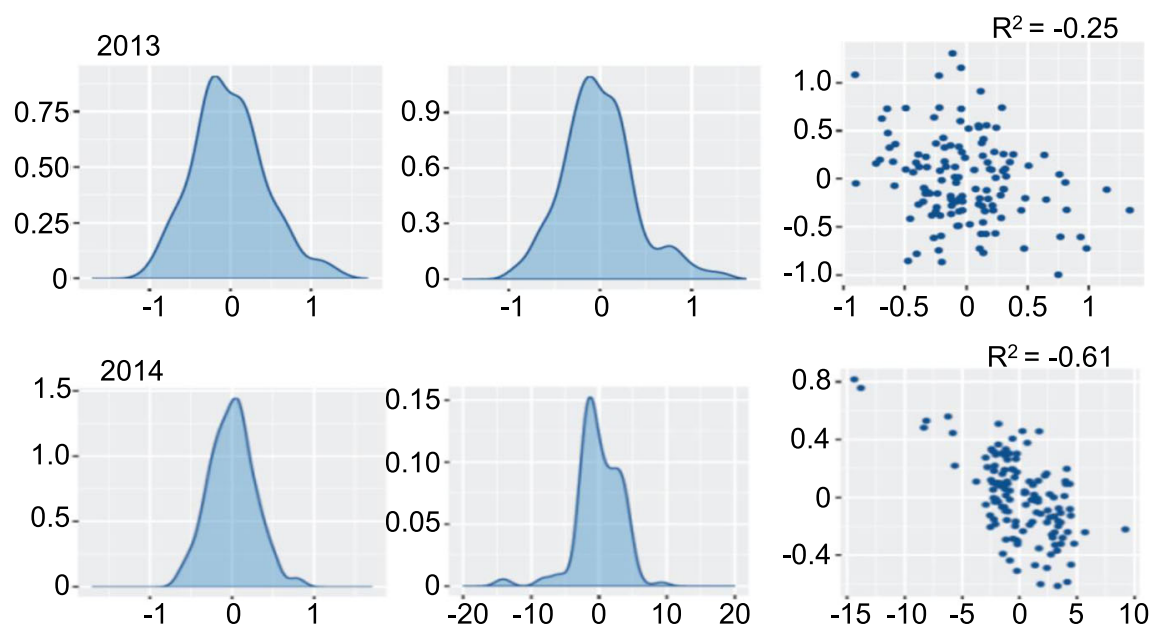

\section{5}
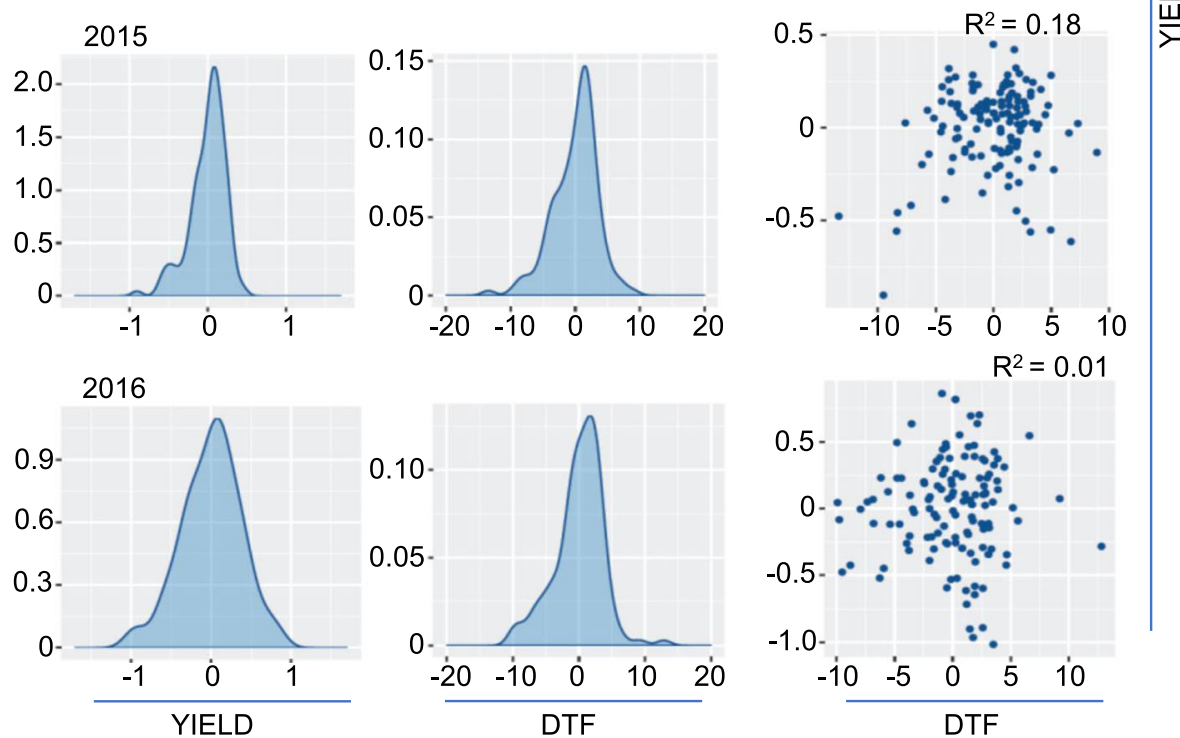

Fig. 6 Distribution of flowering time variation in the biparental mapping population. Pair-plots showing genetic correlation of EBLUPS (empirical best linear unbiased estimators) from the univariate analysis of flowering time and grain yield among 144 doubled haploid lines of $B$. napus population derived from Skipton/Ag-Spectrum//Skipton. DH lines were grown across 4 phenotypic environments (2013-2016) in field plots, 2013 at Euberta, NSW, Australia; 2014 at Wagga Wagga, NSW, Australia [13]), 2015 and 2016 at the Wagga Wagga (this study)

(21 SNPs) located in intron II (Fig. 9). Interestingly, an 8-bp deletion of the sequence 'TTCCGGAA' (coordinates BnC04: $12,437,458-12,437,465 \mathrm{bp})$ was observed in exon-IV of BnC04.FT in seven accessions; Av-Garnet, BC92157, Skipton, Charlton, BLN3614, ATR-Cobbler, ATR-Gem and in Darmor-bzh (reference genotype). This mutation creates a frameshift that removes the highly conserved C-terminal domain containing the PEBP-domain and several substratebinding sites. Cluster analysis showed that all variants formed a distinct cluster (Fig. 10). In the BnA07.FTb (BnaA07g33120D) we identified two indel mutations in the coding region (Fig. 9). The first is a single nucleotide deletion in exon 4 that is heterozygous with the wild type allele in Australian varieties; Av-Garnet, Skipton, Charlton, BC92156, Marnoo, BLN3614, Ag-Castle,
Monty, Maluka, BLN3343-C00402, CB-Telfer, ATRGem, Surpass402, ThunderTT, ATR-Mako, Wesroona and Ag-Spectrum (the remaining lines are homozygous wild-type). The deletion results in a frameshift that affects the final 20 amino acids of the encoded peptide, including the 9 amino acids of the PEBP domain. The second InDel is a 3 base-pair mutation in exon 1 (His60-deletion) that is found in all our sequenced lines. These polymorphisms are consisted with the observed QTLs in the vicinity of FT.

\section{Structural variation in FT promoter region}

We further searched CArG box and other motifs for $F L C, S O C 1, S M Z$ and $C O$ which can potentially bind to repress $F T$ expressions [55] in introns (especially 


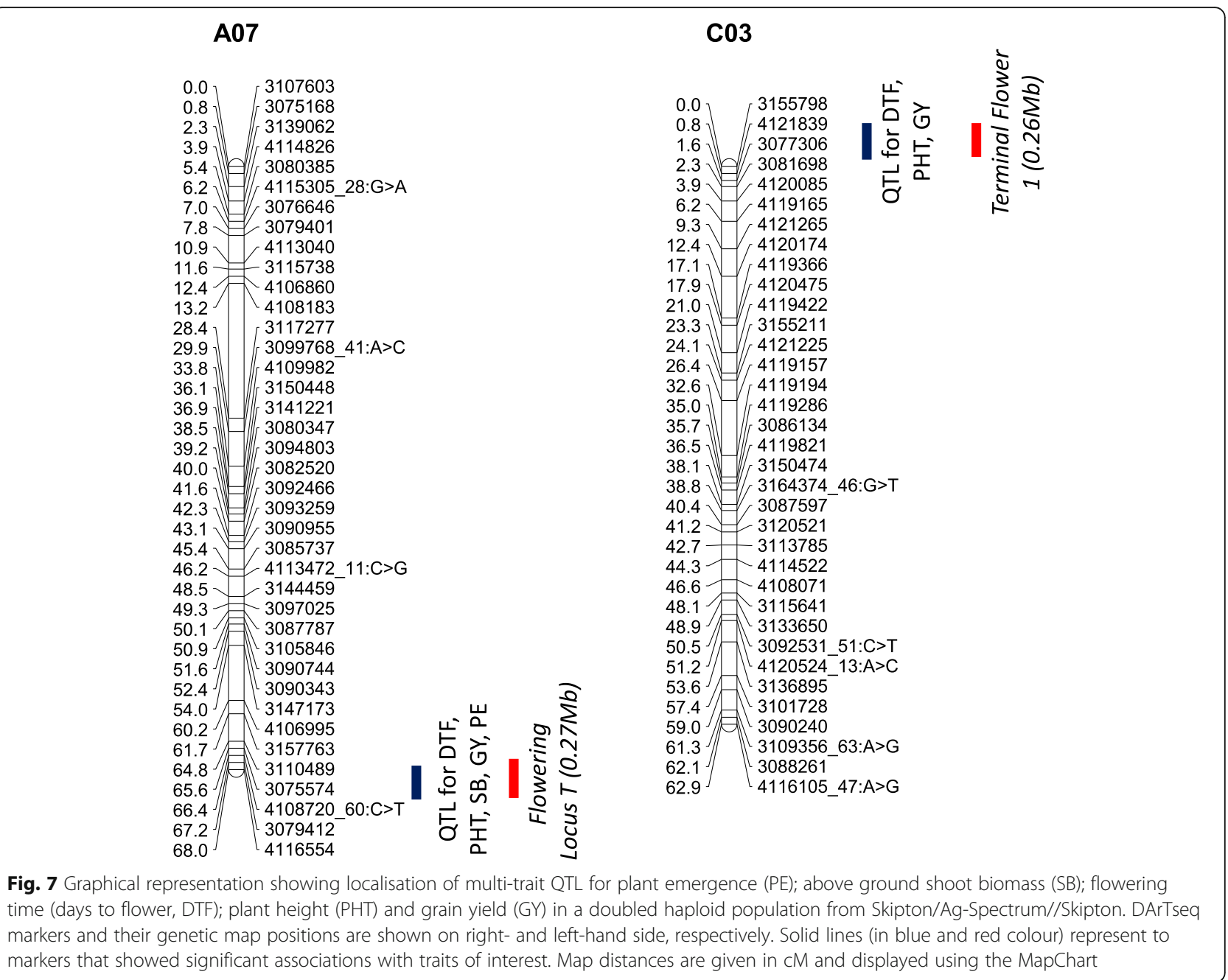

intron 1) exons and promoter regions. A putative $C O$ binding site within Block A: type II = 'ATTGTGGTGATGAGT' (Wang et al. 2009 [24]) was found in both BnA02.FT and BnC02.FT genes. However, this Type-II block 'A' sequence was absent in all FT paralogs located on the A07 and C06 chromosomes. 'CArG' box (CC(A/T)6GG) was absent in introns 1 of $B n A 02 . F T$ and BnC02.FT genes. We also found several 'CACTA' elements in B. napus FT paralogs. For example, in $B n a C 04914850$, a total of four motifs were identified; three were present in introns ( 2 in Intron 2 , antisense direction, and one in sense strand), and one CACTA motif was identified in Exon-IV. In BnA02.FT, a total of 834 CACTA motifs were identified in promoter, intron 1 and exon II. We also identified homologous sequences to $F T$ promoter blocks $\mathrm{C}$ and $\mathrm{E}$ of $A$. thaliana [56] in three $B$. napus $F T$ genes (BnaC06g27090D, BnaA07g25310D, and BnaA02g12130D). Block $E$ was also identified in BnaC06g27090D with blastn (Additional file 26: Figure S8). In comparison to the Block C alignments, the binding regions were not well conserved in Block $E$. The structural variants for the four FT genes were plotted. Finally, in order to determine whether polymorphism in FT directly relates to flowering time variation, we performed phylogenetic analysis of 21 accessions representing GWAS panel and parents of mapping populations being used in the Australian Brassica Germplasm Improvement Program. Our results showed that grouping for both spring and winter types based on FT paralogs was not that distinct (Fig. 10) suggesting that other key flowering genes such as FLC and FRI may have contributed to diversification of these morphotypes [14, 57].

\section{Discussion}

In this study we explored the genetic architecture underlying phenotypic diversity in flowering time, an important trait involved in plant development, adaptation and productivity. Our results demonstrate that there is extensive 


\section{a}

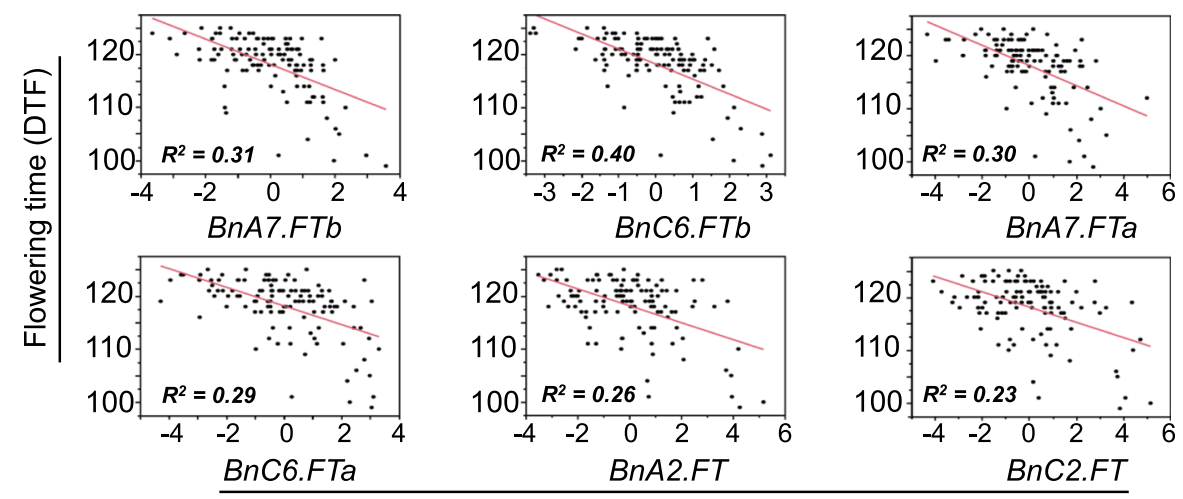

Relative expression levels $F T$ genes $(\ln )$

b

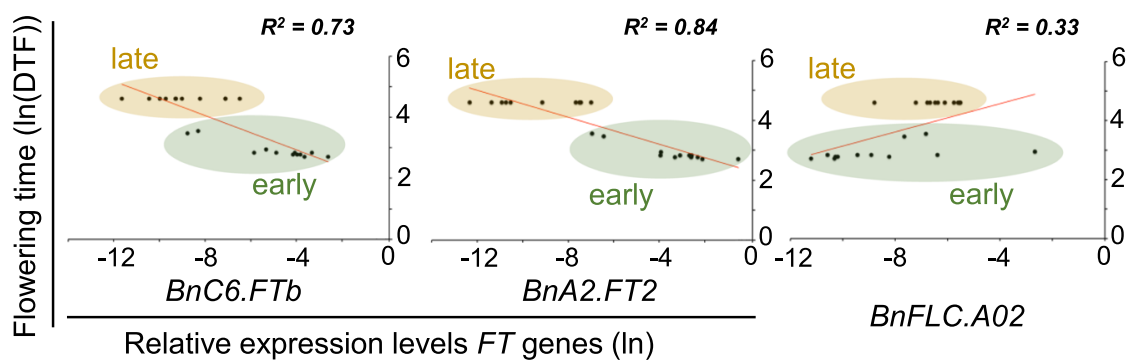

Fig. $8 \mathrm{FT}$ is a major determinant of flowering time variation and photoperiod gene in canola varieties. a Expression analysis of different $F T$ paralogs (BnA2.FTa, BnC2.FT, BnA7.FTa, BnA7.FTb, BnC6.FTa, BnC6.FTb) on leaves taken from field grown plants of 144 doubled haploid lines of Skipton/Ag-Spectrum//Skipton, and its correlation with flowering time. b Expression analyses of FT genes; BnC6.FTb (chromosome C6) and BnA2.FT (chromosome A02) and BnFLC2 on leaves taken from LD grown plants of 24 diverse accessions, representing flowering time diversity in a GWAS panel. The relative expression levels of $F T$ and $F L C$ after normalisation with the reference $U B C 9$, is plotted against flowering time

genetically controlled natural variation in flowering time of canola. Variation in the response to photoperiod (as revealed from $\mathrm{LD}$ and $\mathrm{SD}$ conditions) appears to be another key determinant of flowering time differences among canola accessions (Fig. 1). Despite extended photoperiod at $20^{\circ} \mathrm{C}$, several accessions did not flower under $\mathrm{CE}$ conditions. These accessions flowered when exposed to extended periods of cold temperatures suggesting that these accessions require vernalisation [12, 13, 52]. Thus, a combination of variation in photoperiod and vernalisation response causes phenotypic diversification of flowering time in canola (Fig. 1).

In order to have a minimum effect of vernalisation on flowering time, all field trials were conducted in the middle of June (instead of April the main canola growing season in Australia). We identified a highly significant QTL close to FT locus on chromosome A02 for flowering time variation in field-grown or $\mathrm{CE}$ cabinet-grown plants, suggesting that $F T$ is a major candidate for flowering time across different growing environments (Fig. 4). This QTL was also mapped within $80 \mathrm{~kb}$ of a QTL for vernalisation response in our previous study [12], suggesting that FT integrates signals from both photoperiod and vernalisation pathways and regulates the transition from vegetative to reproductive phase in canola.

The functional role of $F T$ was determined using quantitative RT-PCR using six $F T$ paralog specific primers. Our results demonstrated that all paralogs underlie genetic variation in flowering time in canola. For the first time, we show $F T$ expression in a canola population grown under field conditions is significantly associated with variation in flowering time. It was interesting to observe that most of variation in flowering time was explained by A02 locus in a GWAS panel, and A02 and A07 loci near $F T$ paralogs in the SAgS DH mapping population (Fig. 6, Additional file 12: Table S12). However, the maximum correlation $\left(R^{2}=0.4\right)$ was observed for $B n C 6 . F T b$ homologue, followed by BnA7.FTb $\left(R^{2}=\right.$ 0.31), BnA7.FTa $\left(R^{2}=0.30\right), \quad$ BnC6.FTa $\left(R^{2}=0.29\right)$, $B n A 2 . F T\left(R^{2}=0.26\right)$, and BnC2.FT $\left(R^{2}=0.23\right)$. Higher correlation among different paralogs suggested that different copies can substitute allelic effect on flowering time. Unlike previous studies $[25,26]$, our results suggest that all copies of $F T$ may be functional. Although all FT paralogs except BnC6.FTa and BnC6.FTb map at the same physical position as the closest relative of 


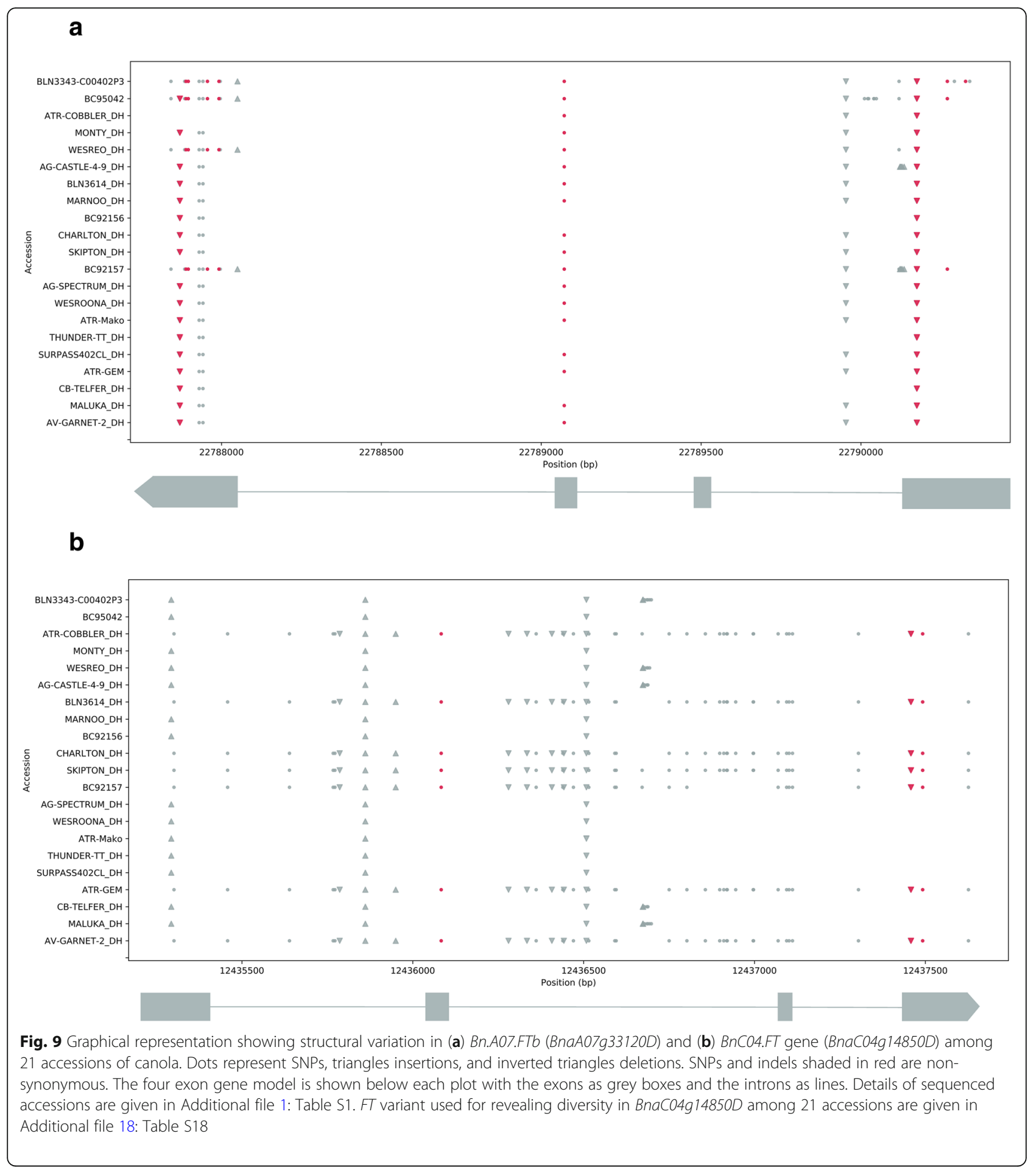

FT, TWIN SISTER OF FT (TSF), cloning of six paralogs of $F T$ in canola [24, 25] discounted the possibility of TSF controlling variation in flowering time which is shown to have much lower expression levels than FT [58-60].

We detect considerable structural variation in promoter, as well as in exonic and intronic regions in $F T$ genes located on chromosomes other than $\mathrm{A} 01$ and $\mathrm{C} 02$. These high levels of polymorphism suggest that the $F T$ gene is a major target for selection during domestication and systematic breeding of canola. $F T$ is a member of the PEBP family and multiple paralogs have evolved from its common ancestral species, however these paralogs may have retained, lost or gained gene function in 


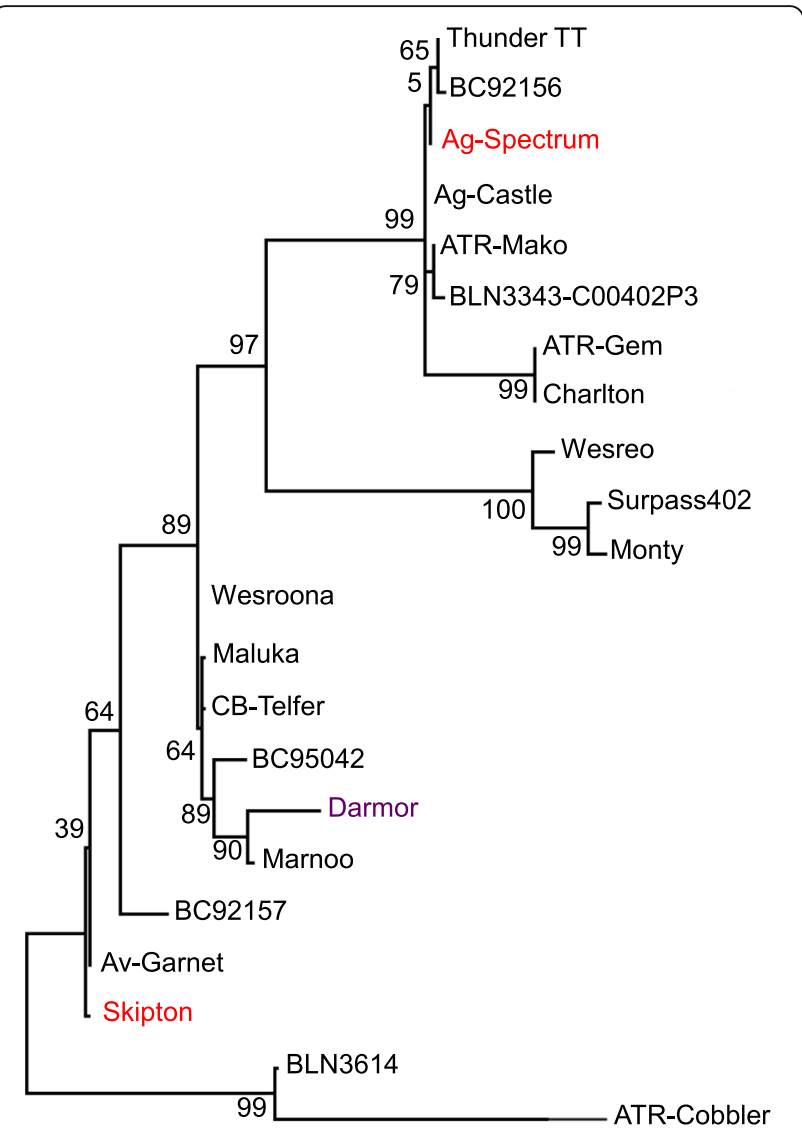

$\stackrel{-1}{0.0005}$

Fig. 10 Neighbour-joining tree based on nucleotide variation across all FT paralogs among 21 accessions of $B$. napus representing GWAS and parental lines (shown in red color) of a doubled haploid population derived from Skipton/Ag-Spectrum//Skipton. Tree was generated in MEGA 6. Nucleotide variation in FT genes was also compared with the corresponding FT genes in the reference Darmor-bzh, in colour. Number refers to percent bootstrap support for branches with greater than $50 \%$ support

the polyploid genome of canola [24, 61]. Our sequencing analyses reveals that different copies of $F T$ harbour mutations including in the CArG, CACTA, Block $C$ and Block $E$ - the binding sites for the transcriptional factors such as FLC, SVP, GI, CO, CIB, CRY2 and SMZ proteins (Additional file 24: Figure S6), which regulate of the expression of $F T$ [1, 25, 56, 62, 63]. Mutations in $F T$ and TFL1 paralogs in canola have been reported to affect flowering and yield components [26]. Mutants or isogenic lines carrying different $F T$ paralogs and/or their combination are required to establish the precise role of each paralog in both vegetative and reproductive phase of plant development. While our expression analyses of FT genes hints at functionality of these paralogs, further research is required to establish whether there is any role of transcriptional enhancers: Block $C$ and Block $E$ on the FT expression [56] as well as its association with other traits of agronomic interest.

We show that FT has multifaceted role in diverse traits that influence plant development. QTLs for several traits such as plant emergence, early vigour, plant biomass, plant height, grain yield, were localized with flowering QTL in a cluster and the expression level of FT showed a good association with different traits. However, this relationship was dependent upon $\mathrm{G} \times \mathrm{E}$ interaction (Additional file 19: Figure S1). These findings hint that flowering time, driven by $F T$ paralogs have variable influence on different traits under different environments. However, it was difficult to establish in this study due to presence of multiple copies of FT in canola genome.

This study demonstrates multigenic inheritance of flowering in the SAgS population. However, a relatively small size population $(n=144)$ may have compromised the estimates of QTL identified herein. In addition, QTL only accounted for small genetic effects (2.7 to $10.3 \%)$ in this study (Additional file 12: Table S12). This is in contrast with other studies, which reported major QTLs for flowering time [64]. Recently, Tyagi et al. [65] showed that Brassica FT homeologs influence flowering time, branching pattern, plant height, silique length and width, seed size, stomatal density, and fatty-acid profile in B. juncea. Our expression analyses revealed that enhanced FT gene expression is related with early flowering in the doubled haploid lines of Skipton/AgSpectrum//Skipton (Fig. 8). In a previous study, Raman et al. [13] showed that early flowering DH lines having Skipton QTL alleles yield higher than those having late flowering allele (Ag-Spectrum). These results suggest that canola varieties having higher $F T$ gene expressions can be selected for enhancing productivity.

In canola, sequence variation in BnFLC.A10 appears to underlie QTL for both flowering time as well as root biomass [21, 66]. In addition, flowering time has been implicated in plasticity of water-use efficiency, carbohydrate availability, plant vigour, resistance to diseases and yield [67-70]. We propose that alleles that showed significant association with flowering time and grain yield in the water-limited years experienced in 2013 and 2014, are of high relevance even though they did not reveal genetic associations in water-unlimited years (non-stress environment, 2015 and 2016). Environmental stress tends to drive changes in flowering time in Brassica as a result of change in allele frequencies of the flowering time genes [71, 72]. Our data also suggest that different FT paralogs regulate flowering time depending upon environment. For example, QTLs for flowering time were identified close to BnaA07g25310D in 2013 and 2014, however a QTL for flowering time was mapped on chromosome C04, close to a different FT paralog, 
BnaC04g14850D in 2015 and 2016. Since, flowering time showed a good correlation with plant emergence, early vigour, shoot biomass, and grain yield; and enhanced $F T$ expression is also correlated with early flowering, it is possible that $F T$ may be one of the drivers promoting early growth in canola, therefore contributing to higher grain yield in canola especially under terminal drought and heat stress environments prevalent in Mediterranean countries.

The findings presented here reveal that the genetic architecture of natural variation in flowering time involves multiple alleles having major effects located near FT, UPSTREAM of FLC and RAV2 paralogs on chromosome A02 (Table 1, Additional file 24: Figure S6). This is in contrast to genetic variation in flowering time regulated by vernalisation which is controlled by multiple alleles distributed across genome [8, 10, 12]. Both positive and negative regulators of $F T$ were located near significant SNP associations; for example, under LD treatment $F L C$ that repress the $F T$ transcription by direct binding to the CArG sites in intron 1 and promoter region of $F T$ was detected [55]. The role of the candidate genes: GI, FD, SAM, $A G L 18 / F U L$ in flowering time is well documented [7]. We also identified significant SNP associations for flowering time in the vicinity of $H^{+}$-ATPse (Additional file 24: Figure S6) which is implicated in stomatal opening and enhanced FT expression in the guard cells [28]. In addition, MSI, $E M F 2, F V E$, and CURLY LEAF which regulate FT transcription via trimethylation of H3K27me3, H3K4me3 and EARLY FLOWERING $6[1,56]$ were located in the vicinity of significant SNPs. These results suggested that both approaches utilized in this study: QTL as well as GWAS analyses are suitable for revealing the genetic architecture of flowering time in canola.

Based on their photoperiodic response, all genotypes could be grouped into photoperiod sensitive, photoperiod insensitive (less sensitive), and non-flowering types (vernalisation sensitive). Classification of such genotypes based on flowering habit was also supported with our molecular marker clustering, which placed the majority of the winter type varieties from Europe, China and Japan, in a single cluster (cluster II, Additional file 21: Fig. S3). These results supported that spring (semispring in Australia), semi-winter and winter canola belong to distinct genepools. A number of semi-winter accessions from China grouped into separate clade. Previous research has shown that Chinese canola germplasm is derived as a result of intensive crossing between winter canola introduced from Europe via Japan and spring type B. rapa for local adaptation [73].

In summary, we have demonstrated through a series of complementary and exploratory analyses based on association tests using genome-wide SNPs, expression QTL and quantitative RT-PCR that the natural variation in flowering time and response to photoperiod revealed in this study is controlled by FT and other loci dispersed across the genome, and modulated by the environment. GWAS approach delineated genomic regions and provided insights into the genetic architecture of flowering time and its multifaceted role in plant development and productivity traits. Although some alleles identified in this study may not be causative of phenotypic differences in flowering time, they still represent valuable selection tools to increase rate of genetic gain in canola improvement programs. Several Illumina Infinium ${ }^{\text {Tx }}$ SNP and $F T$ gene specific markers located near the QTL associated with trait variation and known flowering time genes [74-76] would enable the identification of canola accessions with optimal FT expression and agronomic trait performance. Further research is required to understand the role of different $F T$ copies in canola productivity across target environments.

\section{Additional files}

Additional file 1: Table S1. Accessions used to assess natural variation in flowering time and photoperiodic response. (XLSX 24 kb)

Additional file 2: Table S2. Details for phenotyping, experimental designs and QTL analysis (DOCX $314 \mathrm{~kb}$ )

Additional file 3: Table S3. Mean marker density of Illumina SNP markers genotyped in a canola GWAS panel of 368 accessions. (XLSX 8 kb)

Additional file 4: Table S4. PCR primers used for expression analysis by RT-qPCR (Guo et al. 2014) (XLSX $12 \mathrm{~kb}$ )

Additional file 5: Table S5. Brassica napus genome BLAT HITs against the Arabidopsis thaliana FLOWERING LOCUS T (AT1G65480.1, RSB8/FT/ chr1:24331428-24333935) using Darmor reference assembly (http://www. genoscope.cns.fr/blat-server/cgi-bin/colza/webBlat). FT paralogs identified in a previous study (Schiessl et al. 2014 [45]) are also shown for comparison. (XLSX $28 \mathrm{~kb}$ )

Additional file 6: Table S6. (A) Natural variation in flowering time in a GWAS panel of 368 lines of $B$. napus grown under controlled environment cabinets under short day ( $8 \mathrm{~h}$ light and $16 \mathrm{~h}$ dark) and long day (16 h light and $8 \mathrm{~h}$ dark); (B) Natural variation in flowering time in a GWAS panel of 300 lines of B. napus grown under field conditions. represents to missing data and $(C)$ Broad sense heritability of flowering time under controlled and field condition among canola accessions. (XLSX $8 \mathrm{~kb}$ )

Additional file 7: Table S7. Marker LD across B. napus genome. (CSV $1575 \mathrm{~kb}$ )

Additional file 8: Table S8. Familial relationships between pairs of accessions used for GWAS. (XLSX $41 \mathrm{~kb}$ )

Additional file 9: Table S9. Marker trait association identified for flowering time and photoperiodic response in a GWAS panel of canola. Response to photoperiod was assessed under controlled environment conditions, LD: Long day conditions ( $16 \mathrm{~h}$ light, $8 \mathrm{~h}$ dark at 20 degree); SD ( $8 \mathrm{~h}$ light, $16 \mathrm{~h}$ dark at 20 degree). Flowering time was also evaluated under field conditions at three sites: Wagga Wagga (irrigation, NSW, Australia), Wagga Wagga (lateral move irrigation site) and Condobolin (rainfed site, NSW, Australia) Days to flowering was used for GWAS analysis using GAPIT program in R and Golden Helix (SVS, with and without principal component analysis). (XLSX $9 \mathrm{~kb}$ )

Additional file 10: Table S10. Distribution of significant marker associations for flowering time and photoperiod response, evaluated under controlled environment cabinets and field conditions (three sites) in a GWAS panel of canola (XLSX $936 \mathrm{~kb}$ ) 
Additional file 11: Table S11. Candidate gene associated with flowering time and photoperiodic response in the GWAS and DH population. (XLS $35 \mathrm{~kb}$ )

Additional file 12: Table S12. Significant QTL associated with flowering time and grain yield identified in a doubled haploid population derived from a single $\mathrm{BC}_{1} \mathrm{~F}_{1}$ from the Skipton/Ag-Spectrum//Skipton population grown in four environments, at Euberta (2013) and Wagga Wagga (2014, 2015 and 2016). QTL in bold are repeatedly detected across environments/traits. QTL in bold and italics are multi-trait QTL (pleiotropic). (DOCX $23 \mathrm{~kb}$ )

Additional file 13: Table S13. Genetic correlation between different traits measured in the doubled haploid population from Skipton/AgSpectrum//Skipton across environments. (XLSX $15 \mathrm{~kb}$ )

Additional file 14: Table S14. Genome-wide association analysis (eQTL) showing statistical association between Illumina SNP markers and expression data of BnC6.FT gene in 300 accessions of B. napus. Linear marker regression analysis was performed in the SVS package (Golden Helix). (XLSX $11 \mathrm{~kb}$ )

Additional file 15: Table S15. Gene structures of different $F T$ paralogs identified in the resequence data from 21 accessions of $B$. napus (test samples). Exon/intron genomic coordinates of the B. napus reference cultivar are based on the current gene models (annotation version 5). Numbers in the table represent lengths in base-pairs. Exon/intron length variation in the 21 accessions (in bold) is only counted for InDels that are homozygous. (XLSX $13 \mathrm{~kb}$ )

Additional file 16: Table S16. Summary of structural and polymorphic variation identified among 21 B. napus accessions representing GWAS and validation population used in this study. Numbers in table represent counts of unique variants observed across the 21 accessions. Abbreviations: SNV: structural nucleotide variant, InDel: Insertion-deletion, $\mathrm{S}=$ Number of segregating sites, $\mathrm{ps}=\mathrm{S} / \mathrm{n}, \Theta=\mathrm{ps} / \mathrm{a} 1, \pi=$ nucleotide diversity, and D is the Tajima test statistic (Tajima, 1989). (XLSX $12 \mathrm{~kb}$ )

Additional file 17: Table S17. FT variant used for revealing diversity in BnaA07g33120D among 21 accessions resequenced. (XLSX $10 \mathrm{~kb}$ )

Additional file 18: Table S18. FT variant used for revealing diversity in BnaC04g14850D among 21 accessions resequenced. (XLSX 13 kb)

Additional file 19: Figure S1. Canola genotypes showing $G X E$ interactions when grown under LD and SD conditions in controlled environment cabinet. Mean flowering time is estimated in days. Details of varieties shown here represented to $\mathrm{BC}$ accessions (Additional file 1: Table S1). (PPTX $213 \mathrm{~kb}$ )

Additional file 20: Figure S2. Genome-wide distribution (A) and density (B) of single nucleotide polymorphisms, in a genome wide association diversity panel of 368 Brassica napus accessions. Regions that are rich and poor SNP density are shown in dark and whitehorizontal bars, respectively. The number of SNP markers anchoring on different chromosomes (A1-A10 and (1-C9) of the physical map of the B.napus genome is given on the $\mathrm{x}$-axis. (PPTX $959 \mathrm{~kb}$ )

Additional file 21: Figure S3. Genetic diversity and population structure in a GWAS panel of 368 Brassica napus accessions. Three clusters designated as I, II and III represent predominantly Chinese, European, and Australian accessions, respectively. Details of accessions are given in Additional file 1: Table S1. (PPTX $1670 \mathrm{~kb}$ )

Additional file 22: Figure S4. Principal components (PC1 and PC2) analysis showing population structure in a GWAS diversity panel of 368 B. napus accessions. Three major clusters designated as I, II, and III, consistent with the cluster analysis (Additional file 20: Figure S2). (PPTX $2450 \mathrm{~kb}$ )

Additional file 23: Figure S5. The average linkage disequilibrium (LD) decays $\left(r^{2}\right)$ approach 0.02 when distance between SNPs was approximately $200 \mathrm{~Kb}$. Distance in bp is shown on X-axis. (PPTX $135 \mathrm{~kb}$ )

Additional file 24: Figure S6. Candidate genes located within $200 \mathrm{~kb}$ from the significant SNPs associated with flowering time in a GWAS panel of canola. Accessions were grown under long day (LD, $14 \mathrm{~h}$ light), short day (SD, $8 \mathrm{~h}$ light) treatments in controlled environments (CE) and three field conditions at Wagga Wagga [in single rows: WAG-FT (Row) and plots: WAG-FT (Plots)] and Condobolin [in plots: CON-FT (Plots) Response to photoperiod was estimated as the difference between LD and SD treatments (days). Details are given in Additional file 11: Table S11. (PPTX $189 \mathrm{~kb}$ )

Additional file 25: Figure S7. (A). Frequency distribution of shoot biomass in a SAgS DH population phenotyped across 2014-2016 growing environments. (B). Frequency distribution of fractional ground cover, measured as NSVI with a hand-held GreenSeeker machine, in a SAgS DH population phenotyped across 2015-2016 growing environments). (C). Frequency distribution of days to flower in a SAgS DH population phenotyped across four environments (2013-2016). Phenotypic data of 2013 and 2014 was published previously (Raman et al. 2016 [12, 13, 52]). (D). Frequency distribution of plant height and plant emergence in a SAgS DH population phenotyped in 2016 growing environments. (E). Frequency distribution of grain yield in a SAgS DH population phenotyped across four environments (2013-2016). Phenotypic data of 2013 and 2014 experiments was published previously (Raman et al. 2016 [12, 13, 35]). (PPTX $5970 \mathrm{~kb}$ )

Additional file 26: Figure S8. A: Regions of homology between the B. napus FT regions and block $C$ from $A$, thaliana. Putative binding sites are indicated based on ref. BN_chrC06 is upstream from BnaC06g27090D, BN_chrA07 is upstream from BnaA07g25310D, and BN_chrA02 is upstream from BnaA02g12130D. A corresponding block $C$ region for BnaC02g45250D could not be identified. B: Regions of homology between the $B$. napus FT regions and block E from A. thaliana. Putative binding sites are indicated based on ref. . BN_chrA07 is downstream from BnaA07g25310D, BN chrC02rnd is downstream from BnaC02g45250D, BN_chrA02 is downstream from BnaA02g12130D and BN_chrC06 is downstream from BnaC06g27090D. C: Summary of SNP and Indel variation in the B. napus FT gene BnaA02g12130D across 21 lines. The gene model is shown below the plot. Key: Insertions = triangle, deletions = inverted triangle, SNPS $=$ dots, red = nonsynonymous change. D: Summary of SNP and Indel variation in the B. napus FT gene BnaA07g25310D across 21 lines. The gene model is shown below the plot. Key: Insertions = triangle, deletions = inverted triangle, SNPs = dots, red $=$ nonsynonymous change. E: Summary of SNP and Indel variation in the $B$. napus FT gene BnaC02g45250D across 21 lines. The gene model is shown below the plot. Key: Insertions = triangle, deletions = inverted triangle, SNPs = dots, red = nonsynonymous change. F. Summary of SNP and Indel variation in the B. napus FT gene BnaC06g27090D across 21 lines (only a subset of lines are shown). The gene model is shown below the plot. Key: Insertions = triangle, deletions = inverted triangle, SNPs = dots, red = nonsynonymous change. (PPTX $3860 \mathrm{~kb})$

\section{Abbreviations}

BLAST: Basic local alignment search tool; CE: Controlled environment cabinet; DH: Doubled haploid; eQTL: Expression QTL; FT: Flowering locus T; G X E: Genotype x environment interaction; GWAS: Genome wide association study; MAF: Minor allele frequency; PCR: Polymerase chain reaction; QTL: Quantitative trait loci; SNP: Single nucleotide polymorphism

\section{Acknowledgments}

HR thanks Dr. Bev Orchard NSWDPI for advice on controlled environment experiment designs, Dr. Phil Salisbury (DEDJTR and University of Melbourne) for providing seeds of DHC2211 and DHC2261, Mr. Chris Fuller, Mr. Dean McCallum, and Daryl Reardon (NSW Department of Primary Industries) for carrying-out NDVI, and Kristin Verstermark for help with RNA extractions. HR is thankful to Drs Andrzej Killian, Jie Song and Andrew Kowalczyk at DArT P/ $\mathrm{L}$ for supporting KDCompute pipeline for genetic analyses.

\section{Authors' contributions}

$H R$ conceived the research idea and plans; $H R, R R, Y Q, O O$, and IM carried out the phenotypic experiments; HR and RR conducted genotypic analysis; $L B, R M, R R$ and $H R$ analysed data and carried-out trait-marker associations; HR and RR conducted comparative mapping; ASV, SS, HR and SB performed $F T$ and FLC analyses, HR and DW performed bioinformatics analysis; HR prepared the manuscript and SB revised it. All authors read and commented the manuscript. All authors read and approved the final manuscript. 


\section{Funding}

This work was supported by grants from the Grains Research and Development Corporation (DAN00117, DAN00208) and NSW Agricultural Genomic Centre, BioFirst Initiative of NSW Government, Australia to HR. SS is supported by an ARC-Australian Post-Doctoral Fellowship (DP110100964) and SB is supported by an ARC-Future Fellowship (FT100100377), Larkins Fellowship and a Linkage Development Scheme from Monash University.

\section{Availability of data and materials}

All experimental materials are available on request (email: harsh. raman@dpi.nsw.gov.au). The sequencing data of FT paralogs is given in Additional file 18: Table S18.

\section{Ethics approval and consent to participate}

Not applicable.

\section{Consent for publication}

Not applicable.

\section{Competing interests}

The authors declare that they have no competing interests.

\section{Author details}

${ }^{1} \mathrm{NSW}$ Department of Primary Industries, Wagga Wagga Agricultural Institute, Wagga Wagga, NSW 2650, Australia. ${ }^{2}$ School of Biological Sciences, Monash University, Clayton VIC3800, Australia. ${ }^{3}$ Centre for Bioinformatics and Biometrics, University of Wollongong, Wollongong, NSW 2522, Australia. ${ }^{4} \mathrm{NSW}$ Department of Primary Industries, Orange Agricultural Institute, Orange, NSW 2800, Australia.

\section{Received: 24 February 2019 Accepted: 9 July 2019} Published online: 06 August 2019

\section{References}

1. Pin PA, Nilsson O. The multifaceted roles of FLOWERING LOCUS T in plant development. Plant Cell Environ. 2012;35(10):1742-55.

2. Alonso-Blanco C, Aarts MGM, Bentsink L, Keurentjes JJB, Reymond M, Vreugdenhil D, Koornneef M. What has natural variation taught us about plant development, physiology, and adaptation? Plant Cell. 2009; 21(7):1877-96.

3. Shavrukov $Y$, Kurishbayev A, Jatayev S, Shvidchenko V, Zotova L, Koekemoer F, de Groot S, Soole K, Langridge P. Early flowering as a drought escape mechanism in plants: how can it aid wheat production? Front Plant Sci. 2017:8:1950.

4. Weigel D. Natural variation in Arabidopsis: from molecular genetics to ecological genomics. Plant Physiol. 2012;158(1):2-22.

5. Koornneef M, Alonso-Blanco C, Vreugdenhil D. Naturally occurring genetic variation in Arabidopsis thaliana. Annu Rev Plant Biol. 2004;55:141-72.

6. Bouche F, Lobet G, Tocquin P, Perilleux C. FLOR-ID: an interactive database of flowering-time gene networks in Arabidopsis thaliana. Nucleic Acids Res. 2016;44(D1):D1167-71.

7. Balasubramanian S, Weigel D. Temperature induced flowering in Arabidopsis thaliana. Plant Signal Behav. 2006;1(5):227-8.

8. Raman H, Raman R, Eckermann P, Coombes N, Manoli S, Zou X, Edwards D, Meng J, Prangnell R, Stiller J, et al. Genetic and physical mapping of flowering time loci in canola (Brassica napus L.). Theor Appl Genet. 2013;126:119-32.

9. Nelson MN, Rajasekaran R, Smith A, Chen S, Beeck CP, Siddique KHM, Cowling WA. Quantitative trait loci for thermal time to flowering and photoperiod responsiveness discovered in summer annual-type Brassica napus L. PLoS One. 2014;9(7):e102611.

10. Long Y, Shi J, Qiu D, Li R, Zhang C, Wang J, Hou J, Zhao J, Shi L, Park B-S, et al. Flowering time quantitative trait loci analysis of oilseed brassica in multiple environments and genomewide alignment with Arabidopsis. Genetics. 2007;177(4):2433-44.

11. Ferreira ME, Satagopan J, Yandell BS, Williams PH, Osborn TC. Mapping loci controlling vernalisation requirement and flowering time in Brassica napus. Theor Appl Genet. 1995;90:727-32.

12. Raman H, Raman R, Coombes N, Song J, Prangnell R, Bandaranayake C, Tahira R, Sundaramoorthi V, Killian A, Meng J, et al. Genome-wide association analyses reveal complex genetic architecture underlying natural variation for flowering time in canola. Plant Cell Environ. 2016;39(6):1228-39.
13. Raman R, Diffey S, Carling J, Cowley R, Kilian A, Luckett D, Raman H. Quantitative genetic analysis of yield in an Australian Brassica napus doubled haploid population. Crop Pasture Sci. 2016;67(4):298-307.

14. Schiess S, Iniguez-Luy F, Qian W, Snowdon RJ. Diverse regulatory factors associate with flowering time and yield responses in winter-type Brassica napus. BMC Genomics. 2015;16:737

15. Yi L, Chen C, Yin S, Li H, Li Z, Wang B, King GJ, Wang J, Liu K. Sequence variation and functional analysis of a FRIGIDA orthologue (BnaA3.FRI) in Brassica napus. BMC Plant Biol. 2018;18(1):32

16. Xu L, Hu K, Zhang Z, Guan C, Chen S, Hua W, Li J, Wen J, Yi B, Shen J, et al. Genome-wide association study reveals the genetic architecture of flowering time in rapeseed (Brassica napus L.). DNA Res. 2016;23(1):43-52.

17. Wang N, Qian W, Suppanz I, Wei L, Mao B, Long Y, Meng J, Muller AE, Jung C. Flowering time variation in oilseed rape (Brassica napus L.) is associated with allelic variation in the FRIGIDA homologue BnaA.FRI.a. J Exp Bot. 2011; 62(15):5641-58.

18. Tadege M, Sheldon C, Helliwell C, Stoutjesdijk P, Dennis E, Peacock W. Control of flowering time by FLC orthologues in Brassica napus. Plant J. 2001;28(5):545-53.

19. Hou J, Long Y, Raman H, Zou X, Wang J, Dai S, Xiao Q, Li C, Fan L, Liu B, et al. A Tourist-like MITE insertion in the upstream region of the BnFLC.A10 gene is associated with vernalization requirement in rapeseed (Brassica napus L.). BMC Plant Biol. 2012;12(1):238.

20. Zou X, Suppanz I, Raman H, Hou J, Wang J, Long Y, Jung C, Meng J. Comparative analysis of FLC homologues in Brassicaceae provides insight into their role in the evolution of oilseed rape. PLoS One. 2012;7(9):e45751.

21. Fletcher RS, Mullen JL, Heiliger A, McKay JK. QTL analysis of root morphology, flowering time, and yield reveals trade-offs in response to drought in Brassica napus. J Exp Bot. 2015;66(1):245-56.

22. Koornneef M, Alonso-Blanco C, Blankestijn-de Vries H, Hanhart CJ, Peeters AJM. Genetic interactions among late-flowering mutants of Arabidopsis. Genetics. 1998;148(2):885-92.

23. Koornneef $M$, Hanhart CJ, Veen JH. A genetic and physiological analysis of late flowering mutants in Arabidopsis thaliana. Mol Gen Genet. 1991; 229(1):57-66.

24. Wang J, Long Y, Wu B, Liu J, Jiang C, Shi L, Zhao J, King GJ, Meng J. The evolution of Brassica napus FLOWERING LOCUS T paralogues in the context of inverted chromosomal duplication blocks. BMC Evol Biol. 2009;9:271.

25. Wang J, Hopkins CJ, Hou J, Zou X, Wang C, Long Y, Kurup S, King GJ, Meng $J$. Promoter variation and transcript divergence in Brassicaceae lineages of FLOWERING LOCUS T. PLoS One. 2012;7(10):e47127.

26. Guo Y, Hans H, Christian J, Molina C. Mutations in single FT- and TFL1paralogs of rapeseed (Brassica napus L.) and their impact on flowering time and yield components. Front Plant Sci. 2014;5:282.

27. Navarro C, Abelenda JA, Cruz-Oro E, Cuellar CA, Tamaki S, Silva J, Shimamoto K, Prat S. Control of flowering and storage organ formation in potato by FLOWERING LOCUS T. Nature. 2011;478:119-22.

28. Kinoshita T, Ono N, Hayashi Y, Morimoto S, Nakamura S, Soda M, Kato Y, Ohnishi M, Nakano T, Inoue S, et al. FLOWERING LOCUS T regulates stomatal opening. Curr Biol. 2011;21(14):1232-8.

29. Krieger U, Lippman ZB, Zamir D. The flowering gene SINGLE FLOWER TRUSS drives heterosis for yield in tomato. Nat Genet. 2010;42(5):459-63.

30. Shalit A, Rozman A, Goldshmidt A, Alvarez JP, Bowman JL, Eshed Y, Lifschitz E. The flowering hormone florigen functions as a general systemic regulator of growth and termination. Proc Natl Acad Sci U S A. 2009;106(20):8392-7.

31. Lifschitz E, Eviatar T, Rozman A, Shalit A, Goldshmidt A, Amsellem Z, Alvarez $J P$, Eshed $Y$. The tomato FT ortholog triggers systemic signals that regulate growth and flowering and substitute for diverse environmental stimuli. Proc Natl Acad Sci U S A. 2006;103:6398-403.

32. Lee R, Baldwin S, Kenel F, McCallum J, Macknight R. FLOWERING LOCUS T genes control onion bulb formation and flowering. Nat Commun. 2013;4: 2884. https://doi.org/10.1038/ncomms3884.

33. Cabrera-Bosquet L, Molero G, Stellacci AM, Bort J, Nogues S, Araus J. NDVI as a potential tool for predicting biomass, plant nitrogen content and growth in wheat genotypes subjected to different water and nitrogen conditions. Cereal Res Commun. 2011;39:147-59.

34. Cowley RB, Luckett DJ, Moroni JS, Zeleke K, Diffey S. Remote sensing of early vigour in canola germplasm: relationship to grain yield and potential to select for drought tolerance. Crop Pasture Sci. 2014;65(12): 1288-99. https://doi.org/10.1071/CP14055. 
35. Raman H, Raman R, Coombes N, Song J, Diffey S, Kilian A, Lindbeck K, Barbulescu DM, Batley J, Edwards D, et al. Genome-wide association study identifies new loci for resistance to Leptosphaeria maculans in canola. Front Plant Sci. 2016;7:1513.

36. Rutkoski JE, Poland J, Jannink J-L, Sorrells ME. Imputation of unordered markers and the impact on genomic selection accuracy. G3. 2013;3(3):42739 doi: 410.1534/g1533.1112.005363.

37. Atwell S, Huang YS, Vilhjalmsson BJ, Willems G, Horton M, Li Y, Meng D, Platt A, Tarone AM, Hu TT, et al. Genome-wide association study of 107 phenotypes in Arabidopsis thaliana inbred lines. Nature. 2010; 465(7298):627-31

38. Clarke WE, Higgins EE, Plieske J, Wieseke R, Sidebottom C, Khedikar Y, Batley J, Edwards D, Meng J, Li R, et al. A high-density SNP genotyping array for Brassica napus and its ancestral diploid species based on optimised selection of single-locus markers in the allotetraploid genome. Theor Appl Genet. 2016;129(10):1887-99.

39. Saitou N, Nei M. The neighbor-joining method: a new method for reconstructing phylogenetic trees. Mol Biol Evol. 1987;4(4):406-25.

40. Zhang Z, Ersoz E, Lai C-Q, Todhunter RJ, Tiwari HK, Gore MA, Bradbury PJ, Yu J, Arnett DK, Ordovas JM, et al. Mixed linear model approach adapted for genome-wide association studies. Nat Genet. 2010;42(4):355-60.

41. Kang HM. Efficient control of population structure in model organism association mapping. Genetics. 2008;178:1709-23.

42. Lipka AE, Tian F, Wang Q, Peiffer J, Li M, Bradbury PJ, Gore MA, Buckler ES, Zhang Z. GAPIT: genome association and prediction integrated tool. Bioinformatics. 2012;28(18):2397-9.

43. Team RDC. R: a language and environment for statistical computing. Vienna: R Foundation for Statistical Computing; 2014. ISBN 3-900051-07-0, URL http://www.R-project.org/.

44. Chalhoub B, Denoeud F, Liu S, Parkin IAP, Tang H, Wang X, Chiquet J, Belcram $\mathrm{H}$, Tong C, Samans B, et al. Early allopolyploid evolution in the post-Neolithic Brassica napus oilseed genome. Science. 2014;345(6199):950-53.

45. Raman H, Raman R, Diffey S, Qiu Y, McVittie B, Barbulescu DM, Salisbury PA, Marcroft S, Delourme R. Stable quantitative resistance loci to blackleg disease in canola (Brassica napus L.) over continents. Front Plant Sci. 2018; 91622. doi: https://doi.org/10.3389/fpls.2018.01622.

46. Schiessl S, Samans B, Hüttel B, Reinhardt R, Snowdon RJ. Capturing sequence variation among flowering-time regulatory gene homologues in the allopolyploid crop species Brassica napus. Front Plant Sci. 2014;5.

47. Edgar RC. MUSCLE: multiple sequence alignment with high accuracy and high throughput. Nucleic Acids Res. 2004;32(5):1792-7.

48. Wang K, Li M, Hakonarson H. ANNOVAR: functional annotation of genetic variants from next-generation sequencing data. Nucleic Acids Res. 2010:38:e164.

49. Tamura K, Stecher G, Peterson D, Filipski A, Kumar S. MEGA6: Molecular Evolutionary Genetics Analysis version 6.0. Mol Biol Evol. 2013;30:2725-9.

50. Tajima F. Statistical methods to test for nucleotide mutation hypothesis by DNA polymorphism. Genetics. 1989;123:585-95.

51. Fay JC, Wu Cl. Hitchhiking under positive Darwinian selection. Genetics. 2000;155(3):1405-13

52. Raman R, Taylor B, Marcroft S, Stiller J, Eckermann P, Coombes N, Rehman A, Lindbeck K, Luckett D, Wratten N, et al. Molecular mapping of qualitative and quantitative loci for resistance to Leptosphaeria maculans; causing blackleg disease in canola (Brassica napus L.). Theor Appl Genet. 2012;125(2): 405-18.

53. Luckett DJ, Cowley R, Moroni S, Raman H. Improving water-use efficiency and drought tolerance in canola - potential contribution from improved carbon isotope discrimination (CID). In: Proceedings of the 13th International Rapeseed Congress, Prague; 2011.

54. Tollenaere R, Hayward A, Dalton-Morgan J, Campbell E, Lee JRM, Lorenc M, Manoli S, Stiller J, Raman R, Raman H, et al. Identification and characterization of candidate R/m4 blackleg resistance genes in Brassica napus using next-generation sequencing. Plant Biotechnol J. 2012;10(6): 709-15.

55. Deng W, Ying H, Helliwell CA, Taylor JM, Peacock WJ, Dennis ES. FLOWERING LOCUS C (FLC) regulates development pathways throughout the life cycle of Arabidopsis. Proc Natl Acad Sci. 2011;108(16):6680-5.

56. Adrian J, Farrona S, Reimer JJ, Albani MC, Coupland G, Turck F. cisRegulatory elements and chromatin state coordinately control temporal and spatial expression of FLOWERING LOCUS T in Arabidopsis. Plant Cell. 2010;22(5):1425-40.
57. Schiessl S, Huettel B, Kuehn D, Reinhardt R, Snowdon R. Postpolyploidisation morphotype diversification associates with gene copy number variation. Sci Rep. 2017;7:41845

58. Michaels SD, Himelblau E, Kim SY, Schomburg FM, Amasino RM. Integration of flowering signals in winter-annual Arabidopsis. Plant Physiol. 2005;137(1):149-56.

59. Yamaguchi A, Kobayashi Y, Goto K, Abe M, Araki T. TWIN SISTER OF FT (TSF) acts as a floral pathway integrator redundantly with FT. Plant Cell Physiol. 2005;46(8):1175-89.

60. Jang S, Torti S, Coupland G. Genetic and spatial interactions between FT, TSF and SVP during the early stages of floral induction in Arabidopsis. Plant J. 2009;60(4):614-25

61. Chalhoub B, Denoeud F, Liu SY, Parkin IAP, Tang HB, Wang XY, Chiquet J, Belcram H, Tong CB, Samans B. Early allopolyploid evolution in the postNeolithic Brassica napus oilseed genome. Science. 2014:345.

62. Helliwell CA, Wood CC, Robertson M, James Peacock W, Dennis ES. The Arabidopsis FLC protein interacts directly in vivo with SOC1 and FT chromatin and is part of a high-molecular-weight protein complex. Plant J. 2006;46(2):183-92

63. Bouche F, Lobet G, Tocquin P, Perilleux C. FLOR-ID: an interactive database of flowering-time gene networks in Arabidopsis thaliana. Nucleic Acids Res. 2016:44.

64. Osborn TC, Kole C, Parkin IAP, Sharpe AG, Kuiper M, Lydiate DJ, Trick M. Comparison of flowering time genes in Brassica rapa, B. napus and Arabidopsis thaliana. Genetics. 1997;146(3):1123-9.

65. Tyagi S, Mazumdar PA, Mayee P, Shivaraj SM, Anand S, Singh A, Madhurantakam C, Sharma P, Das S, Kumar A, et al. Natural variation in Brassica FT homeologs influences multiple agronomic traits including flowering time, silique shape, oil profile, stomatal morphology and plant height in B. juncea. Plant Sci. 2018;277:251-66.

66. Fletcher RS, Herrmann D, Mullen JL, Li Q, Schrider DR, Price N, Lin J, Grogan K, Kern A, McKay JK. Identification of polymorphisms associated with drought adaptation QTL in Brassica napus by resequencing. G3. 2016;6(4): 793-803.

67. Graf A, Schlereth A, Stitt M, Smith AM. Circadian control of carbohydrate availability for growth in Arabidopsis plants at night. Proc Natl Acad Sci. 2010;107(20):9458-63.

68. Kenney AM, McKay JK, Richards JH, Juenger TE. Direct and indirect selection on flowering time, water-use efficiency (WUE, $\delta 13 C$ ), and WUE plasticity to drought in Arabidopsis thaliana. Ecol Evol. 2014;4(23):4505-21.

69. Ni Z, Kim E-D, Ha M, Lackey E, Liu J, Zhang Y, Sun Q, Chen ZJ. Altered circadian rhythms regulate growth vigour in hybrids and allopolyploids. Nature. 2009;457(7227):327-31.

70. Wei D, Mei J, Fu Y, Disi J, Li J, Qian W. Quantitative trait loci analyses for resistance to Sclerotinia sclerotiorum and flowering time in Brassica napus. Mol Breed. 2014;34(4):1797-804.

71. Franks SJ, Kane NC, O'Hara NB, Tittes S, Rest JS. Rapid genome-wide evolution in Brassica rapa populations following drought revealed by sequencing of ancestral and descendant gene pools. Mol Ecol. 2016;25(15): 3622-31.

72. Franks SJ, Sim S, Weis AE. Rapid evolution of flowering time by an annual plant in response to a climate fluctuation. Proc Natl Acad Sci U S A. 2007; 104(4):1278-82

73. Qian W, Meng J, Li M, Frauen M, Sass O, Noack J, Jung C. Introgression of genomic components from Chinese Brassica rapa contributes to widening the genetic diversity in rapeseed (B. napus L.), with emphasis on the evolution of Chinese rapeseed. Theor Appl Genet. 2006:113:49-54.

74. Bernier G, Perilleux C. A physiological overview of the genetics of flowering time control. Plant Biotechnol J. 2005:3(1):3-16.

75. Dennis ES, Peacock WJ. Epigenetic regulation of flowering. Curr Opin Plant Biol. 2007:10(5):520-7.

76. Michaels SD. Flowering time regulation produces much fruit. Curr Opin Plant Biol. 2009;12:75-80.

\section{Publisher's Note}

Springer Nature remains neutral with regard to jurisdictional claims in published maps and institutional affiliations. 